\title{
CHIANTI - an atomic database for emission lines
}

\section{Wavelengths greater than $50 \AA^{\star}$}

\author{
K.P. Dere ${ }^{1}$, E. Landi ${ }^{2}$, H.E. Mason ${ }^{3}$, B.C. Monsignori Fossi ${ }^{4}$, and P.R. Young ${ }^{3}$ \\ 1 Naval Research Laboratory, Washington DC, U.S.A. \\ 2 Department of Astronomy and Space Science, University of Florence, Italy \\ 3 Department of Applied Mathematics and Theoretical Physics, University of Cambridge, UK \\ 4 Arcetri Astrophysical Observatory, Florence, Italy
}

Received July 24; accepted November 5, 1996

\begin{abstract}
A comprehensive set of accurate atomic data is required for analyses of astrophysical and solar spectra. CHIANTI provides a database of atomic energy levels, wavelengths, radiative data and electron excitation data for ions which are abundant in cosmic plasmas. The most recent electron excitation data have been assessed and stored following the method of Burgess \& Tully (1992). The current version is essentially complete for specifying the emission spectrum at wavelengths greater than $50 \AA$. A list of observed lines in the spectral region between 50 and $1100 \AA$ has been compiled and compared with the lines predicted by the CHIANTI database. The CHIANTI database reproduces the vast majority of lines observed at these wavelengths. CHIANTI includes IDL (Interactive Data Language) routines to calculate optically thin synthetic spectra for equilibrium conditions. IDL routines to calculate theoretical line intensities required for electron density or temperature diagnostics and emission measure studies are also included. The CHIANTI atomic database and supporting IDL routines are available by anonymous FTP.
\end{abstract}

Key words: atomic data — astronomical data bases: miscellaneous - ultraviolet: general - Sun: atmosphere - stars: atmosphere

\section{Introduction}

Many astrophysical emission line spectra are produced by the collisional excitation of a variety of ionization stages

Send offprint requests to: K.P. Dere

* Table 3 only available in electronic form at CDS via anonymous ftp to cdsarc.u.strasbg.fr $(130.79 .128 .5)$ or via http://cdsweb.u-strasbg.fr/Abstract.html of elements commonly found in cosmic plasmas. Their interpretation requires an accurate knowledge of the atomic structure of these ions and their excitation and emission rates (Mason \& Monsignori Fossi 1994). In the past a number of spectral codes have been constructed to aid in the interpretation of astrophysical spectra. Examples include those of Landini \& Monsignori Fossi (1970, 1990), Tucker \& Koren (1971), Mewe (1972), Mewe \& Gronenschild (1981), Mewe et al. (1985), Kato (1976), Raymond \& Smith (1977), Stern et al. (1978), and Gaetz \& Salpeter (1983). A comparison and critique of different plasma emission codes for X-ray and UV spectra is published by Mason (1996a). Many of these codes were assembled at a time when the necessary atomic data such collision strengths were often lacking. Often the $\bar{g}$ formula (Van Regemorter 1962), or variations, were used to estimate electron excitation rates. Because of the increasing power of computer technology, reliable calculations of many of these excitation rates are continually becoming available.

Recent papers have attempted to provide more accurate representations for the atomic parameters, in particular for the iron ions (cf. Brickhouse et al. 1995; Monsignori Fossi \& Landini 1994a; Mewe et al. 1995).

The basic goal for the CHIANTI database is to construct a database that includes the best available calculations of atomic parameters for analyzing astrophysical emission line spectra. In implementing the database, we also tried to achieve several other goals: 1 . the database could be readily updated, 2 . the database would be easy to distribute, 3 . it would be transparent to the end user, 4. accuracy would be maintained by visually examining as much of the input data as possible, 5 . it would use a data and programming structure that would facilitate the development of programs by end users.

The basic unit of the data base is the individual ion. For each ion there is a directory that contains a file specifying its energy levels, a file providing wavelengths and 
radiative rates, and a file providing fits to the collision strengths which give the electron excitation rates. Each file contains information on the source of the data and other relevant comments. The energy level information is largely taken from the NIST database of observed energy levels (Martin et al. 1995), updated by more recent observed values and supplemented by our best theoretical estimates where energy levels are not known. Radiative data are obtained from available publications and supplemented where necessary by new calculations. Collision strengths $(\Omega$ 's) and upsilons ( $\Upsilon$ 's - collision strengths averaged over a Maxwellian velocity distribution) have all been visually inspected and scaled according to the formulation of Burgess \& Tully (1992). Elemental abundances can be freely specified and the ionization equilibrium is determined from steady state calculations, for which the Arnaud \& Rothenflug (1985) calculation is supplied.

At the present time, the database is capable of reproducing the optically thin emission line spectrum at wavelengths greater than $50 \AA$ for electron densities less than about $10^{15} \mathrm{~cm}^{-3}$. The ions necessary to calculate the spectrum below $50 \AA$ will be developed in the near future. There is no long wavelength limit to the database but, in a practical sense, it becomes less comprehensive at longer wavelengths because neutrals are not included. The scaling of the upsilons should be accurately reproducible for nearly any range of temperature. Astrophysically abundant elements from hydrogen through nickel are included. Tables 1 and 2 show which ions are currently included in the CHIANTI database.

A package of programs written in Interactive Data Language (IDL) are also supplied. These allow the calculation of level populations, synthetic spectra, and density and temperature sensitive line ratios. Because of the nature of the database, further capabilities and interfaces to other programming languages, such as Fortran or C, can be readily implemented in the future. The package is freely available for downloading over the internet. The CHIANTI database and accompanying IDL routines have been incorporated into the scientific analysis software for the Coronal Diagnostic Spectrometer onboard the Solar and Heliospheric Observatory (SOHO) by C.D. Pike and G. Del Zanna.

\section{Emission line intensities}

The emissivity (power per unit volume, $\operatorname{erg} \mathrm{cm}^{-3} \mathrm{~s}^{-1}$ ) for an optically thin spectral line at wavelength $\lambda_{i, j}$ is given by:

$\epsilon_{i, j}=N_{j}\left(X^{+m}\right) A_{j, i} \frac{h c}{\lambda_{i, j}}$

where $A_{j, i}\left(\mathrm{~s}^{-1}\right)$ is the Einstein spontaneous emission coefficient ( $A$ value); $N_{\mathrm{e}}$ is the electron number density $\left(\mathrm{cm}^{-3}\right) ; N_{j}\left(X^{+m}\right)$ is the number density of the level $j$ of the ion $X^{+m}$ :

$N_{j}\left(X^{+m}\right)=\frac{N_{j}\left(X^{+m}\right)}{N\left(X^{+m}\right)} \frac{N\left(X^{+m}\right)}{N(X)} \frac{N(X)}{N(H)} \frac{N(H)}{N_{\mathrm{e}}} N_{\mathrm{e}}$

where $\frac{N j\left(X^{+m}\right)}{N\left(X^{+m}\right)}$ is the population of level $j$ relative to the total $N\left(X^{+m}\right)$ number density of ion $X^{+m}$ and is a function of the electron temperature and density; $\frac{N\left(X^{+m}\right)}{N(X)}$ is the ionization ratio of ion $X^{+m}$ which is predominantly a function of temperature; $\frac{N(X)}{N(H)}$ is the element abundance relative to hydrogen which varies in different astrophysical plasmas and also in different solar features; $\frac{N(H)}{N_{\mathrm{e}}}$ is the hydrogen abundance relative to electron density $(\sim 0.8)$.

The flux at the Earth $\left(\mathrm{erg} \mathrm{cm}^{-2} \mathrm{~s}^{-1}\right)$ in a spectral line is given by:

$I\left(\lambda_{i, j}\right)=\frac{1}{4 \pi R^{2}} \int_{V} \epsilon_{i, j} \mathrm{~d} V$

where $V$ is the volume of emission and $R$ is the earth-toobject distance.

In low density plasmas the collisional excitation processes are generally faster than ionization and recombination timescales, therefore the collisional excitation is dominant over ionization and recombination in populating excited states. The low lying level populations can then be treated separately from the ionization and recombination processes.

The number density population of level $j$ must be calculated by solving the statistical equilibrium equations for a number of low lying levels and including all the important collisional and radiative excitation and de-excitation mechanisms:

$$
\begin{aligned}
& N_{j}\left(N_{\mathrm{e}} \Sigma_{i} C_{j, i}^{\mathrm{e}}+N_{\mathrm{p}} \Sigma_{i} C_{j, i}^{\mathrm{p}}+\Sigma_{i>j} R_{j, i}+\Sigma_{i<j} A_{j, i}\right)= \\
& \Sigma_{i} N_{i}\left(N_{\mathrm{e}} C_{i, j}^{\mathrm{e}}+N_{\mathrm{p}} C_{i, j}^{\mathrm{p}}\right)+\Sigma_{i>j} N_{i} A_{i, j}+\Sigma_{i<j} N_{i} R_{i, j}
\end{aligned}
$$

with $C_{j, i}^{\mathrm{e}}$ and $C_{j, i}^{\mathrm{p}}$ the electron and proton collisional excitation rate coefficients $\left(\mathrm{cm}^{3} \mathrm{~s}^{-1}\right), R_{j, i}$ the stimulated absorption rate coefficient $\left(\mathrm{s}^{-1}\right)$ and $A_{j, i}$ the spontaneous radiation transition probability $\left(\mathrm{s}^{-1}\right)$.

In this release of CHIANTI, we do not include proton excitation rates, which are important for fine structure transitions in highly ionized systems. An extensive review of theoretical work, together with recommended data has been compiled by Copeland et al. (1996).

The ratios of emission line intensities provide a multitude of spectroscopic diagnostics for determining the plasma parameters. Extensive reviews have recently been provided by Mason \& Monsignori Fossi (1994), Dwivedi (1994) and Doschek (1997), together with a very useful bibliography by Keenan (1996). 
Table 1. Ions included in the CHIANTI database

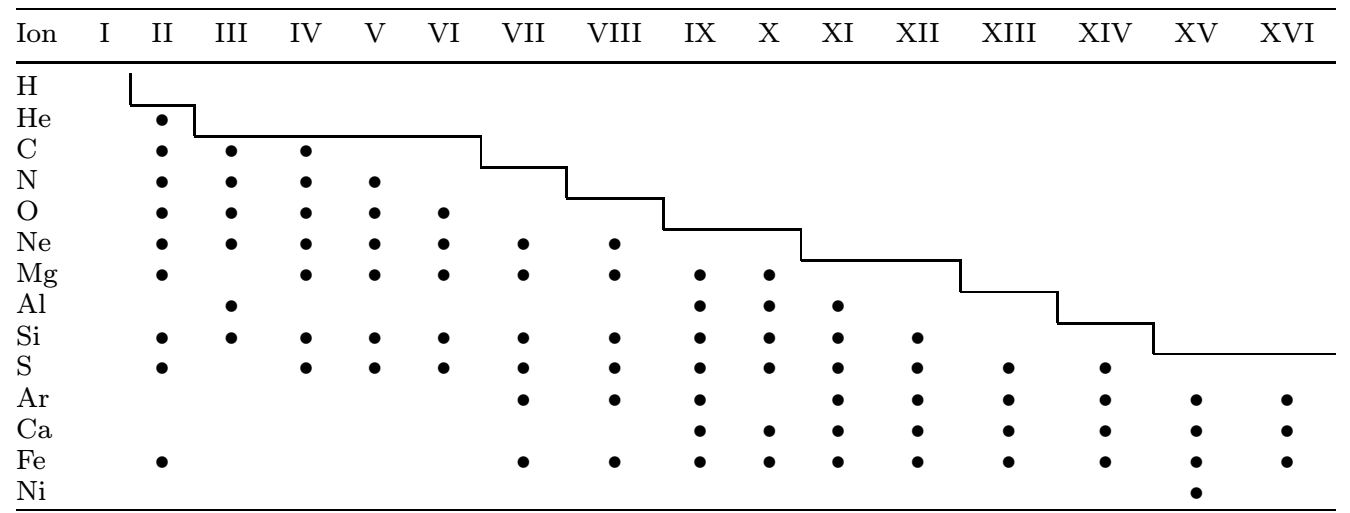

Table 2. Ions included in the CHIANTI database

\begin{tabular}{|c|c|c|c|c|c|c|c|c|c|c|c|c|}
\hline Ion & XVII & XVIII & XIX & XX & XXI & XXII & XXIII & XXIV & XXV & XXVI & XXVII & XXVIII \\
\hline $\begin{array}{l}\mathrm{Ar} \\
\mathrm{Ca}\end{array}$ & $\bullet$ & • & & & & & & & & & & \\
\hline $\mathrm{Fe}$ & $\bullet$ & $\bullet$ & $\bullet$ & • & $\bullet$ & $\bullet$ & $\bullet$ & $\bullet$ & & & & \\
\hline $\mathrm{Ni}$ & $\bullet$ & $\bullet$ & $\bullet$ & $\bullet$ & & & & $\bullet$ & $\bullet$ & $\bullet$ & & \\
\hline
\end{tabular}

\section{Atomic data}

\subsection{Energy levels}

In general the energy levels are taken from the NIST (Martin et al. 1995) database. For those levels not available from the NIST database we have calculated theoretical energies using the UCL SSTRUCT program (Eissner et al. 1974). These have been carefully adjusted to provide what we consider to be our "best estimates" of the predicted energy values for individual ions. In those cases where there is no reliable energy, the energy is taken from the target values in the atomic scattering calculation.

The energy levels are generally arranged so that the higher energy levels correspond to a higher index. However, we have found that it is often more useful to use a fixed ordering for the LSJ levels throughout an isoelectronic sequence in order to aid in data evaluation and in data interpolation and extrapolation.

\subsection{Radiative data}

For each ion, one file contains the radiative data including wavelengths, weighted oscillator strengths and $A$ values. The wavelengths are calculated from the energy levels based on observed spectra. If values of the observed energy levels are not available, the wavelength is calculated from the theoretical wavelength but is stored in the file as a negative value to distinguish reliable wavelengths from predicted wavelengths. All wavelengths are vacuum wave- lengths. Radiative transition probabilities ( $A$ values) are generally taken from the literature. Transitions for which the branching ratio is less than $10^{-5}$ have been removed from the files that are generally distributed but are still available.

In a number of cases, the radiative data are not available in the literature and we have used the radiative code SSTRUCT, described in Eissner et al. (1974), to calculate theoretical energy levels and radiative data. A scaled central potential is used to obtain the one-electron radial wavefunctions and the scaling parameters are obtained by minimizing the term energies. Using the code is relatively simple - the main inputs being the charge of the ion and the configurations to be used in the model-however some care has to be taken in the choice of configurations to ensure the accuracy of the energy levels and radiative transition probabilities.

SSTRUCT can calculate electric dipole (E1) and quadrupole (E2), and magnetic dipole (M1) and quadrupole (M2) transition probabilities. For Chianti, the code has been used for three main cases: ions for which no electronic version of the radiative data exists - in such cases the SSTRUCT data was checked against the original data, e.g., Fe X; ions for which the previously calculated radiative data could be improved upon, e.g., Fe IX; individual ion transitions for which radiative data was unavailable, e.g., Fe XIII. Where SSTRUCT has been used, 
the configurations used in the calculation are documented in the radiative data files.

\subsection{Electron collisional data}

The electron collisional excitation rate coefficient $\left(\mathrm{cm}^{3}\right.$ $\mathrm{s}^{-1}$ ) for a Maxwellian electron velocity distribution with a temperature $T_{\mathrm{e}}(\mathrm{K})$, is given by:

$C_{i, j}^{\mathrm{e}}=\frac{8.6310^{-6}}{T_{\mathrm{e}}^{1 / 2}} \frac{\Upsilon_{i, j}\left(T_{\mathrm{e}}\right)}{\omega_{i}} \exp \left(\frac{-E_{i, j}}{k T_{\mathrm{e}}}\right)$

where $\omega_{i}$ is the statistical weight of level $i ; E_{i, j}$ is the energy difference between levels $i$ and $j ; k$ is the Boltzmann constant and $\Upsilon_{i, j}$ is the thermally-averaged collision strength:

$\Upsilon_{i, j}\left(T_{\mathrm{e}}\right)=\int_{0}^{\infty} \Omega_{i, j} \exp \left(-\frac{E_{j}}{k T_{\mathrm{e}}}\right) \mathrm{d}\left(\frac{E_{j}}{k T_{\mathrm{e}}}\right)$

where $E_{j}$ is the energy of the scattered electron relative to the final energy state of the ion. The collision strength $\Omega$ is a symmetric $\left(\Omega_{i, j}=\Omega_{j, i}\right)$, dimensionless quantity. It is related to the electron excitation crosssection $\sigma_{i, j}=\pi a_{0}^{2}\left(\Omega_{i, j} / \omega_{i}\right) / E$, where $\pi a_{0}^{2}$ is the area of the first Bohr orbit and $E$ is the incident electron energy in Rydbergs. The electron de-excitation rates are obtained by the principle of detailed balance.

Collision strengths are obtained from theoretical calculations. The solution of the electron-ion scattering problem is complex and requires extensive computing resources. The accuracy of a particular calculation depends on two main factors. The first is the representation which is used for the target wavefunctions, the second is the type of scattering approximation chosen.

The target must take account of configuration interaction and allow for intermediate coupling for the higher stages of ionization. The main approximations used for electron-ion scattering are Distorted Wave (DW), Coulomb Bethe $(\mathrm{CBe})$ and the more elaborate CloseCoupling (CC) approximation. The DW approximation neglects the coupling of the channels (target + scattering electron). Since the scattering electron sees a central field potential, the DW approximation is valid for systems which are more than a few times ionized. The University College London (UCL) DW program (Eissner \& Seaton 1972) has been extensively used by Nussbaumer, Flower, Mason, Bhatia and co-workers. For high partial wave values of the incoming electron, the CBe approximation (Burgess \& Shoerey 1974) is valid, when it is assumed that the scattering electron does not penetrate the target.

The Coulomb Born (CB) approximation has been used extensively in early work. This takes no account of the distortion of the scattering electron's wavefunction due to the central field potential. The Coulomb Born Oppenheimer (CBO) approximation includes exchange (cf. Burgess et al. 1970).
Extreme care must be taken to ensure that the high partial wave contribution is accurately accounted for in both dipole and non-dipole transitions. This has not always been the situation in published results. The Burgess \& Tully (1992) scaling method allows one to determine whether or not the electron scattering calculations are tending towards their correct high energy or temperature limits. Burgess et al. (1996) use the Coulomb Born approximation to determine the high energy limits for non-dipole transitions.

In the CC approximation, the scattering electron sees individual target electrons, the channels are coupled and a set of integro-differential equations are solved. A CC code which has been extensively used is the R-matrix package (Burke et al. 1971; Berrington et al. 1978) developed at Queens University of Belfast (QUB). The CC approximation is the most accurate. It is also the most expensive in terms of computing resources and it has sometimes been necessary to truncate the size of the target, i.e. the number of interacting configurations which are included. Sometimes pseudo states (non-spectroscopic orbitals) are used to simulate the missing configurations. In practice, the accuracy of any calculation depends on the target as well as the scattering approximation, so it should not automatically be assumed that $\mathrm{CC}$ results are always better than DW results. In general DW is thought to be accurate to about $25 \%$ and CC to better than $10 \%$. The agreement between CC and DW calculations for allowed transitions is usually excellent (Burgess et al. 1989, 1991). Resonance structures can contribute significantly to the excitation rates, particularly for forbidden and intersystem lines and these generally can be accounted for only by the CC models. Although resonances can contribute significantly to the excitation rates, the process of radiation damping can reduce their effect for highly ionized systems. This importance of this process has only recently been acknowledged and allowing for it is still not straightforward.

It is important that electron scattering calculations for highly ionized systems take account of the spin-orbit effect and other relativistic corrections to the target wavefunctions. For low stages of ionization, the transformation from LS coupling to intermediate coupling (IC) is straightforward - it is just algebraic. However, for higher stages of ionization, the transformation from LS to IC must take account of the relevant relativistic interactions as a perturbation to the non-relativistic Hamiltonian. The scattering calculations are often carried out with target in LS coupling, including some one-body terms, then the target is transformed using term coupling coefficients (TCC) from an atomic structure calculation in IC coupling (cf. (Saraph 1972).

A relativistic DW code has been developed by Sampson, Zhang and co-workers (Zhang \& Sampson 1994) based on the multi-configuration Dirac-Fock program of Grant and co-workers (Grant et al. 1980). It does not calculate the resonance structure or allow for the coupling 
between channels. A Dirac-Fock R-matrix (DARC) package has been developed by Norrington \& Grant (1987). Comparisons of collision data between the Dirac-Fock Rmatrix, relativistic distorted wave, Breit-Pauli R-matrix, and R-matrix with TCC's is generally good for the iron ions and elements with $Z \leq 26$.

Recent advances in laboratory techniques have enabled more accurate determinations of the electron excitation rate coefficients for ions. This field of research is too extensive to cover in this paper but excellent reviews are given by Henry (1993) and Dunn (1992).

A semi-empirical formula commonly used in astrophysics is the effective Gaunt factor or $\bar{g}$ approximation (Van Regemorter 1962). This is based on the CBe approximation and relates the collision strength to the oscillator strength for electric dipole transitions:

$\Omega_{i, j}=\frac{8 \pi}{\sqrt{3}} \omega_{j} f_{i, j} \frac{I_{\mathrm{H}}}{E_{i, j}} \bar{g}$.

With this formula, it is possible to estimate an electron excitation rate when only the oscillator strength is known.

Younger \& Wiese (1979) assessed the reliability of the effective Gaunt factor approximation. They found that for $\Delta n=0$ transitions in alkali-like ions (where $n$ is the principal quantum number), the Gaunt factor is within $25 \%$ of unity and varies slowly with energy. They proposed an approximate expression for the Gaunt factor as a function of $Z$ and incident energy for the $\Delta n=0$ case. For the case $\Delta n \neq 0$, they found that the Gaunt factor varies from 0.05 to 0.7 as a function of energy and concluded that it was not possible to produce a reliable approximation to the effective Gaunt factor. They also conclude that the effective Gaunt factor approximation is unsuitable for forbidden and intercombination transitions. These often have resonances in the collision strength, which are quite narrow in energy and numerous so that they can make a substantial contribution to the excitation rate in addition to the nonresonant component. More recently, Sampson \& Zhang (1992) have re-assessed the use of the Van Regemorter formula. They found it to be frequently a very poor approximation, especially for $\Delta n \geq 1$ excitation transitions and recommended that with the present availability of accurate atomic data, the use of the Van Regemorter formula should be abandoned.

Mewe (1972) and Mewe \& Gronenschild (1981) abandoned the simple Van Regemorter formula and introduced a polynomial with four terms $A_{n} E^{-n}$ and a logarithmic term $A_{5} \log E$ expressing the electron energy $(E)$ dependence of the electron excitation Gaunt factor. This expression can be integrated over a Maxwellian electron energy distribution. The coefficients $A_{n}$ can be adjusted to fit both calculated collision strengths and measured excitation rates as well as to estimate collision strengths when only few or even no calculations exist. The inclusion of the $\log E$ term provides the capability of having the correct functional form at high electron impact energies.
3.4. Assessment of electron excitation data

Considerable effort has recently been put into the assessment of published atomic data, in particular electron excitation rates. A workshop on this topic was held in Abingdon in March 1992 sponsored by the SOHO CDS and SUMER projects. The proceedings, edited by J. Lang, are published as a single volume of the Atomic Data and Nuclear Data Tables (Lang 1994). Review papers are presented on each of the isoelectronic sequences from H-like to Ne-like, together with several Si and S ions and Fe IFe XXVI. These reviews critically assess published data and provide tables or graphs of recommended $\Upsilon$ values. This work together with the bibliography of Itikawa (1991) and the compilation by Pradhan \& Gallagher (1992) provide a comprehensive survey of the electron excitation rates required for spectral diagnostics of astrophysical plasmas. In the meantime, new calculations of $\Omega$ 's and $\Upsilon$ 's continue to become available.

The ions of iron pose a difficult challenge for electron scattering calculations, because of the complexity required to accurately represent the $n=3$ configurations. Mason (1994) has assessed available publications for the coronal ions Fe IX-Fe XIV. She found that existing electron excitation data are severely limited in accuracy. Subsequently, new data for Fe X and Fe XI have been calculated by Bhatia \& Doschek (1995a, 1996), which are used here. However, there still remain serious inadequacies with the current atomic data and detailed work using the $\mathrm{CC}$ approximation and very accurate targets (including relativistic effects) is underway as part of the Iron Project (Hummer et al. 1993) to remedy this situation. We intend to incorporate these results in future releases of CHIANTI.

Electron excitation data are provided by authors in many different formats. Some give $\Omega$ as a function of energy, others give $\Upsilon$ as a function of temperature. Our task has been to gather these electron excitation data together, to assess it and to present it in a compressed and easily accessible format. Burgess \& Tully (1992) provide a very useful method for critically examining the electron scattering data and highlighting differences between calculations. The method is based on scaling the incident electron energy and the collision strength so that they both fall within a given, finite range. Each "type" of transition (ie electric dipole allowed, forbidden, intercombination) is treated differently. Taking the case of dipole (allowed) transitions as an example, the formulation for the scaled energy, $x$, is given by $x=1-\frac{\ln (C)}{\ln (X-1+C)}$ and the scaled collision strength, $y$, by $y=\Omega \ln (X-1+\mathrm{e})$ where $X$ is the colliding electron energy in threshold units and $C$ is a variable which can be adjusted to suit the case. The scaled energy $x$ varies between 0 (threshold) and 1 (infinite energy). The high energy limit for $\Omega$ and high temperature limit for $\Upsilon$ is obtained from the Coulomb Bethe approximation; it is simply $4 \omega_{i} f_{i, j} / E_{i, j}$, where $\omega_{i} f_{i, j}$ is 
the weighted dipole oscillator strength and $E_{i, j}$ is the energy difference in Ryd.

Burgess \& Tully's interactive graphical programs are written in BBC BASIC. They are not very portable and cannot be run on Unix workstations, although they can be run on PC type machines with an emulator. In this work we have developed IDL routines based on the Burgess \& Tully (1992) concept and methods, called BURLY (from BURgess..tulLY). An important aspect of scaling collision strengths by the Burgess and Tully approach is that the scaled values can often be approximated by a straight line so that the extrapolation of the collision strength to threshold and to infinite energy becomes relatively straightforward. A knowledge of the collision strength over the full energy range is needed to determine the integration over a Maxwellian velocity distribution which is carried out with a Gauss-Laguerre method. The collision strengths are finally expressed as a 5 point spline fit to the scaled collision strengths. Burgess et al. (1997) have developed a method for obtaining the infinite energy or temperature values of the collisional data from the Coulomb Born approximation. It is hoped that this will be incorporated into our fitting programs in a future release. We considered it to be very important to ensure that all of the collisional data, each ion and transition, should be visually examined.

The use of the Burgess and Tully scaling has revealed several problems with the calculations of $\Omega$ 's and $\Upsilon$ 's. The version of the UCL DW code that has been used until recently for most of the DW calculations requires that the energy of the incident electron be higher than the energy of the highest level of the target ion. Consequently, many DW wave calculations provide values of $\Omega$ at energies considerably above the threshold so that extrapolation of the calculated points back to the threshold can be uncertain. The value at the threshold often dominates in determining the average over a Maxwellian, especially at low temperature. As pointed out by Burgess et al. (1991) and Burgess \& Tully (1992), a potential problem with collision strength calculations is the tendency to include an insufficient number of partial waves, often because of their cost in terms of computing resources. The higher partial wave contributions are estimated from the Coulomb Bethe or Coulomb Born approximations or using a geometrical progression. Inadequate account of the high partial wave contribution can lead to either an overestimate or underestimate of the collision strength for allowed transitions, particularly at high incident energies, so that the collision strengths do not approach the correct high energy limit given by the Bethe approximation. This can occur with either DW or CC calculations. Examples of this have been uncovered and have been dealt with by truncating the published values at some energy or temperature and then interpolating to the high energy limit.

\section{The CHIANTI database}

The goal of the CHIANTI database is to reproduce the emission spectrum of astrophysical sources at wavelengths greater than about $1 \AA$. This initial version was developed with the expectation that it would be essentially complete for reproducing the emission line spectra at wavelengths above $50 \AA$. This was done in order to meet the immediate needs for analyses of astrophysical spectra obtained by experiments such as EUVE, Hubble, and SOHO. Consequently, ions in the helium isoelectronic sequence have not been included and He II is the only ion in the hydrogen isoelectronic sequence to be included in the current version. We expect to include these ions in the near future. Many transitions at wavelengths shorter than $50 \AA$ are included in the database but it is not complete at the shorter wavelengths.

\subsection{The hydrogen isoelectronic sequences: He II}

For He II the 25 fine structure levels of the $1 \mathrm{~s}, 2 l, 3 l, 4 l$, and $5 l$ configurations have been included. Observed energies are from Kelly (1987). Radiative constants ( $g f$ and $A$ values) were taken from Wiese et al. (1966) for dipole transitions. For the $1 \mathrm{~s}{ }^{2} \mathrm{~S}_{1 / 2}-2 \mathrm{~s}^{2} \mathrm{~S}_{1 / 2}$ magnetic dipole and two photon electric dipole transitions, the $A$ values of Parpia \& Johnson (1972) are used.

Aggarwal et al. (1991b) have performed R-matrix calculations of collisions strengths for transitions among the $n=1-5$ levels of He II (i.e. $1 \mathrm{~s}, 2 \mathrm{~s}, 2 \mathrm{p}, 3 \mathrm{~s}, 3 \mathrm{p}, 3 \mathrm{~d}, 4 \mathrm{~s}, 4 \mathrm{p}$, 4d, 4f, 5s, 5p, 5d, 5f, and 5g). Aggarwal et al. (1992) noted that the 1991 calculations were performed at energies up to 7 Ryd and that the collision strengths in the intermediate energy region above those energies where the threshold resonances occur are overestimates of the actual collision strength. Consequently, they combined the close-coupling collision strengths of Unnikrishnan et al. (1991) in the 4.4 to 14.71 Ryd energy range with the collision strengths of Aggarwal et al. (1991b) in the threshold region to arrive at better estimates of the collision strengths to the $2 \mathrm{~s}, 2 \mathrm{p}$, $3 \mathrm{~s}, 3 \mathrm{p}$, and $3 \mathrm{~d}$ levels. We have used these values. For the $n=4$ and $n=5$ levels, we have used the original calculations of Aggarwal et al. (1991b). To distribute the collision strengths between 2 LS states among the fine structure levels, we have scaled the collision strengths following the rules for the distribution of $g f$ values under LS coupling.

\subsection{The lithium isoelectronic sequence}

The atomic structure of the lithium-like ions is relatively simple. Since there are no intersystem transitions, the calculation of collision rates and radiative data is relatively straightforward. The consequent lack of metastable levels means that density sensitive line ratios are not available but the interpretation of lithium sequence line intensities in terms of emission measures is simplified. The $2 \mathrm{~s}-2 \mathrm{p}$ transitions are quite strong and provide good diagnostics 
over a range of temperatures, from C IV in the transition regions, through $\mathrm{Mg} \mathrm{X}$ and $\mathrm{Si} \mathrm{XII}$ in the quiet corona and to Fe XXIV in flares. The electron excitation data available for the lithium-like ions seems to be very accurate (McWhirter 1994).

The lithium-like ions provide an opportunity for a precise comparison between laboratory measurements of electron excitation rate coefficients $(2 \mathrm{~s}-2 \mathrm{p})$ and theoretical work. The recent laboratory measurements for C IV, N $\mathrm{V}$ and O VI agree well with theory, to within the typical uncertainties (20\%) quoted. However, there remains a long-standing discrepancy for Be II.

Also, they provide an opportunity for determining the electron temperature in the solar atmosphere using the intensity ratios of the $2 \mathrm{~s}-2 \mathrm{p}$ relative to $2 \mathrm{~s}-3 \mathrm{p}$ transitions (McWhirter 1976). In particular, the O VI ratio $1032 \AA / 173 \AA$ is a potentially useful diagnostic for SOHO (Bely-Dubau 1994).

\subsubsection{IV and N V}

For the lithium isoelectronic sequence ions $\mathrm{C} \mathrm{IV}$ and $\mathrm{N} \mathrm{V}$, the configurations $2 \mathrm{~s}^{2} 2 \mathrm{~s}, 2 \mathrm{~s}^{2} 2 \mathrm{p}, 2 \mathrm{~s}^{2} 3 \mathrm{~s}, 2 \mathrm{~s}^{2} 3 \mathrm{p}$, and $2 \mathrm{~s}^{2} 3 \mathrm{~d}$ were included. Values for the observed energy levels were taken from Kelly (1987). Oscillator strengths and spontaneous transition probabilities ( $A$ values) were taken from Wiese et al. (1966) and Martin et al. (1993). Gau \& Henry (1977) provided analytical fits to their calculations of the necessary collision strengths for several ions in the lithium isoelectronic sequence and these have been used here. Their functional form $\left(\Omega=c_{0}+c_{1} / X+c_{2} \ln X\right)$ provides the correct high energy behavior $(\sim \ln X)$ but it has been necessary to modify their parameter $c_{2}$ to ensure an exact match to the Coulomb-Bethe high energy limit.

Laboratory measurements of the absolute electron excitation rate coefficients for the transition $2 \mathrm{~s}-2 \mathrm{p}$ in C IV have been carried out by Savin et al. (1995). These measurements agree well with the 9 state $\mathrm{CC}$ calculations of Burke (1992).

\subsubsection{O VI, Ne VIII, Mg X, Al XI, Si XII, S XIV, Ar XVI, Ca XVIII, Fe XXIV and Ni XXVI}

Observed energies for the 24 fine structure levels of $2 l, 3 l$, $4 l, 5 l$ configurations for O VI, Ne VIII and Ar XVI are obtained from Kelly (1987) and for Mg X, Al XI, Si XII, S XIV, Ca XVIII, Fe XXIV, and Ni XXVI are obtained from the NIST database.

Zhang et al. (1990) have calculated relativistic distorted wave collision strengths for the the lithium isoelectronic sequence from O VI through Ni XXVI. In addition, they provide oscillator strengths from which the radiative data has been calculated. Some additional oscillator strengths come from Martin et al. (1993).

As an example of the Burgess and Tully collision strength scaling, the data for the $\mathrm{Mg} X \mathrm{~s}^{2} 2 \mathrm{~s}^{2} \mathrm{~S}_{1 / 2}$ to
$1 \mathrm{~s}^{2} 3 \mathrm{p}^{2} \mathrm{P}_{3 / 2}$ dipole transition is shown in Fig. 1 . The spline fits to the scaled $\Upsilon$ 's show an average deviation of about $0.2 \%$.

\subsection{The beryllium isoelectronic sequence}

The beryllium-like ions contain metastable levels $(2 \mathrm{~s} 2 \mathrm{p}$ ${ }^{3} \mathrm{P}_{0,1,2}$ in the first excited configuration. Since the early work with Skylab, these ions have provided useful electron density diagnostics for the solar transition region (cf. Gabriel \& Jordan 1972; Dupree et al. 1976; Dere \& Mason 1981). In particular the diagnostic potential of the C III lines has been extensively explored (Doschek 1997).

A comparison of the laboratory and theoretical transition probabilities for the resonance and intercombination lines in the Be-like sequence has recently been published by Ralchenko \& Vainshtein (1996). Although on the whole the agreement is good, there still remain some discrepancies to resolve.

The available electron excitation rates for the beryllium-like ions was reviewed by Berrington (1994). For the low ion stages, it is important to include the channel coupling and resonance effects of the $n=3$ states on the $n=2$ to $n=2$ transitions. R-matrix calculations are therefore recommended. For the highly ionized systems the CC and DW results are found to be in good agreement.

\subsubsection{III}

The adopted atomic model for C III includes 6 configurations: $2 \mathrm{~s}^{2}, 2 \mathrm{~s} 2 \mathrm{p}, 2 \mathrm{p}^{2}, 2 \mathrm{~s} 3 \mathrm{~s}, 2 \mathrm{~s} 3 \mathrm{p}$ and $2 \mathrm{~s} 3 \mathrm{~d}$, corresponding to 20 fine structure levels. Experimental energies from the NIST database are available for all levels, and they have been used to calculate transition wavelengths. Radiative transition probabilities for all possible transitions between the 20 levels have been taken from Bhatia \& Kastner (1992). No radiative transition probability for the level is $2 \mathrm{~s} 2 \mathrm{p}^{3} \mathrm{P}_{0}$ is available in the literature. The decay would involve a forbidden $J=0$ to $J=0$ transition to the ground level. Collisional data for transitions between $2 \mathrm{~s}^{2}, 2 \mathrm{~s} 2 \mathrm{p}$ and $2 \mathrm{p}^{2}$ levels have been taken from the R-Matrix calculations of Berrington et al. (1985). Maxwellian averaged collision strengths are calculated for 9 values of electron temperature in the range $10^{4}-10^{6} \mathrm{~K}$. Adopted collisional data for transitions from $n=2$ to $n=3$ and between $n=3$ levels are reported in Berrington et al. (1989): they provide R-Matrix effective collision strengths for 10 values of electron temperature. Both R-Matrix calculations adopt the LS coupling scheme for all levels. In order to transform to intermediate coupling their thermal averaged collision strengths have been scaled using the statistical weights of the levels. 

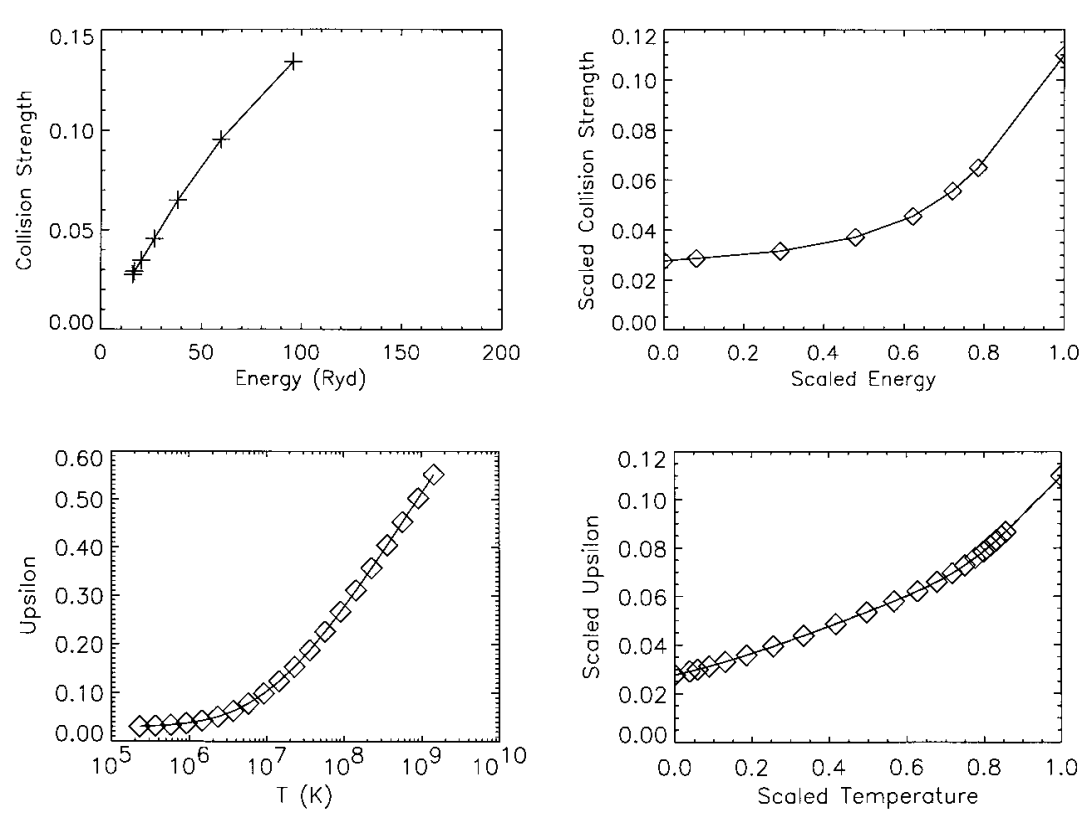

Fig. 1. Collision strengths (Zhang et al. 1990) for $\mathrm{MgX} 1 \mathrm{~s}^{2} 2 \mathrm{~s}^{2} \mathrm{~S}_{1 / 2}-1 \mathrm{~s}^{2} 3 \mathrm{p}^{2} \mathrm{P}_{3 / 2}$. Upper left, the original collision strengths, upper right, the scaled collision strengths with the high energy limit from the Bethe approximation, lower left, the values of $\Upsilon$, the collision strengths convolved with a Maxwellian over the energy range from threshold to infinity, and, lower right, the scaled values of $\Upsilon$ following the Burgess \& Tully (1992) scaling for an allowed transition

\subsubsection{N IV}

The adopted atomic model for N IV includes 6 different configurations: $2 \mathrm{~s}^{2}, 2 \mathrm{~s} 2 \mathrm{p}, 2 \mathrm{p}^{2}, 2 \mathrm{~s} 3 \mathrm{~s}, 2 \mathrm{~s} 3 \mathrm{p}$ and $2 \mathrm{~s} 3 \mathrm{~d}$, corresponding to 20 fine-structure energy levels. Experimental energy levels come from the NIST database and have been used to calculate all transition wavelengths. Theoretical energy levels come from Ramsbottom et al. (1994). A values and oscillator strengths come from the unpublished calculations of Bhatia and are available for all possible transitions between the 20 energy levels. Again, no radiative transition probability is available for $2 \mathrm{~s} 2 \mathrm{p}{ }^{3} \mathrm{P}_{0}$ level. Maxwellian averaged collision strengths are taken from the R-Matrix calculations of Ramsbottom et al. (1994). $\Upsilon$ values are provided for 31 values of electron temperature for all transitions between the 20 fine structure levels in the range $10^{3}-10^{6} \mathrm{~K}$.

For some transitions from Ramsbottom et al. the behavior of the effective collision strengths as a function of electron temperature is quite complex and the 5-point spline interpolation technique is not able to reproduce with the required accuracy their dependence on $T_{\mathrm{e}}$. For this reason the values of the effective collision strengths for temperatures lower than $10^{3.6} \mathrm{~K}$ have been omitted from the CHIANTI database, so that the interpolation technique could reproduce adequately the behavior of the curve. This omission of points should not have any consequence for studies of transition region and coronal plasmas where the temperature is much higher than $10^{3.6} \mathrm{~K}$ and the temperature of maximum abundance for $\mathrm{N}$ IV is $\simeq 10^{5.5} \mathrm{~K}$.

\section{4. $O \mathrm{~V}$}

For $\mathrm{O} \mathrm{V}$, the configurations $2 \mathrm{~s}^{2}, 2 \mathrm{~s} 2 \mathrm{p}, 2 \mathrm{p}^{2}, 2 \mathrm{~s} 3 \mathrm{~s}, 2 \mathrm{~s} 3 \mathrm{p}$ and 2s3d are included. Energy levels from the NIST database are available for all of the 20 fine structure levels. Zhang \& Sampson (1992) provide electric dipole oscillator strengths and $A$ values for all allowed transitions. Additional radiative transition probabilities are taken from Muhlethaler \& Nussbaumer (1976) for forbidden and intercombination transitions. For transitions from the $n=3$ levels, the $A$ values of Hibbert (1980) have been used.

Zhang \& Sampson (1992) also provide relativistic DW collision strengths among the $n=2$ levels. Collision strengths to the $n=3$ levels are provided by Kato et al. (1990) who use the R-matrix calculations of Berrington \& Kingston (1990). The values of Kato et al. have been recently updated (Kato 1996).

\subsubsection{Ne VII, Mg IX, Si XI}

For these members of the beryllium-like sequence, the configurations $2 \mathrm{~s}^{2}, 2 \mathrm{~s} 2 \mathrm{p}, 2 \mathrm{p}^{2}, 2 \mathrm{~s} 3 \mathrm{~s}, 2 \mathrm{~s} 3 \mathrm{p}, 2 \mathrm{~s} 3 \mathrm{~d}, 2 \mathrm{p} 3 \mathrm{~s}, 2 \mathrm{p} 3 \mathrm{p}$ and 2p3d are included. Energy levels from the NIST database are available for many but not all of the 46 fine structure levels. The number of unidentified levels increases with $Z$. Zhang \& Sampson (1992) provide electric dipole 
oscillator strengths and $A$ values for all allowed transitions. Additional radiative transition probabilities are taken from Muhlethaler \& Nussbaumer (1976) for forbidden and intercombination transitions. Zhang \& Sampson (1992) also provide relativistic DW collision strengths among the $n=2$ levels. The $n=2$ to $n=3$ transition probabilities and Coulomb-Born-exchange collision strengths are taken from Sampson et al. (1984).

We note that R-matrix electron impact excitation rates for Ne VII transitions up to the $n=3$ configurations have recently been published by Ramsbottom et al. $(1994,1995)$. These will be assessed in the next release of CHIANTI.

\subsubsection{Al X, S XIII, Ar XV, Ca XVII, Ni XXV}

The configurations $2 \mathrm{~s}^{2}, 2 \mathrm{~s} 2 \mathrm{p}$ and $2 \mathrm{p}^{2}$, are included. Energy levels from the NIST database are available for all of the 10 fine structure levels. Zhang \& Sampson (1992) provide electric dipole oscillator strengths and $A$ values for all allowed

transitions. Additional radiative transition probabilities are taken from Muhlethaler \& Nussbaumer (1976) for forbidden and intercombination transitions which have been interpolated for Al X and Ar XV. Zhang \& Sampson (1992) also provide relativistic DW collision strengths among the $n=2$ levels. For S XIII and higher, transitions from the $n=3$ levels occur at wavelengths shorter than $50 \AA$. The $n=3$ levels for these ions have not been included in the present version of the CHIANTI database since the additional levels incur a penalty in computational speed when solving for level populations. Nevertheless, they will be included in future releases of CHIANTI.

Berrington (1994) found very good agreement between the DW results for Ca XVII (Bhatia \& Mason 1983) and the R-matrix calculations by Dufton et al. (1983c), even for quite low temperatures. This gives us confidence in the adoption of relativistic DW calculations for the highly ionized Be-like ions.

\subsubsection{Fe XXIII}

The adopted atomic model for Fe XXIII includes 9 configurations, $2 \mathrm{~s}^{2}, 2 \mathrm{~s} 2 \mathrm{p}, 2 \mathrm{p}^{2}, 2 \mathrm{~s} 3 l$ and $2 \mathrm{~s} 4 l$ corresponding to 30 fine structure energy levels. Experimental energies come from the NIST database and have been used to calculate the transition wavelengths. When no experimental energy level was available, the theoretical value has been used. Theoretical energies for configurations $2 \mathrm{~s}^{2}, 2 \mathrm{~s} 2 \mathrm{p}$ and $2 \mathrm{p}^{2}$ come from Zhang \& Sampson (1992). Theoretical values for the remaining configurations, oscillator strengths and $A$ values come from the unpublished calculations of Bhatia. Radiative transition probabilities are available for all transitions between the levels of the adopted atomic model.
Adopted collision strengths for transitions between levels belonging to $2 \mathrm{~s}^{2}, 2 \mathrm{~s} 2 \mathrm{p}$ and $2 \mathrm{p}^{2}$ configurations come from Zhang \& Sampson (1992) (see previous subsection). Collision strengths to the $3 l$ and $4 l$ come from the unpublished distorted wave calculations of Bhatia (1996). Collision strengths are provided for 3 values of the incident electron energy at 115, 345 and 695 Rydberg for all transitions between the 30 levels.

The $\Upsilon$ 's from Zhang \& Sampson have been compared with those determined from the earlier work by Bhatia \& Mason $(1981,1986)$. On the whole the agreement is found to be excellent at around $10^{7} \mathrm{~K}$. However, for a few (non-dipole) transitions, the high temperature behaviour differs. This could be due to lack of convergence for the high partial waves in Bhatia and Mason's calculations. Berrington (1994) found good agreement between the Fe XXIII distorted wave calculations (Bhatia \& Mason 1986) and the sophisticated, fully relativistic (Dirac) R-matrix calculations (Norrington \& Grant 1987; Keenan et al. 1993). The calculations of Bhatia and Mason do not contain the $2 \mathrm{p} 3 l$ and $2 \mathrm{p} 4 l$ configurations. This places a severe limitation on the accuracy of the collision strengths for some of the $n=2$ to $n=3,4$ transitions. This problem is discussed by Bhatia \& Mason (1981).

\subsection{The boron isoelectronic sequence}

The boron isoelectronic sequence has proven a rich source for diagnostic ratios in the solar atmosphere (cf. Vernazza \& Mason 1978). For the low ion stages, transitions from the metastable ${ }^{4} \mathrm{P}_{\mathrm{J}}$ levels in the excited configuration $2 \mathrm{~s} 2 \mathrm{p}^{2}$, which fall at around $1400 \AA$, have been used as a primary diagnostic for measuring the electron pressure in the transition region (cf. Dere et al. 1982). For the coronal ions (Mg VIII, Si X, A XIV, Ca XVI), the relative change in population in the ground levels $-2 \mathrm{~s}^{2} 2 \mathrm{p}^{2} \mathrm{P}_{\mathrm{J}}$ is reflected in the intensities of the UV transitions from the excited configuration $2 \mathrm{~s} 2 \mathrm{p}^{2}$. The intensity ratios of the UV spectral lines have been used to determine electron densities in solar flares (cf. Dere et al. 1979) and other solar features (see references in Dwivedi 1994 and Keenan 1996). X-ray and XUV lines from the ion Fe XXII arise from the transitions between the excited configurations $2 \mathrm{~s} 2 \mathrm{p}^{2}, 2 \mathrm{~s}^{2} 31$ and $2 \mathrm{~s}^{2} 41$ and the ground configuration (Mason \& Storey 1980). These have been recorded in spectra of solar flares and more recently in astrophysical sources (Dupree et al. 1993; Monsignori et al. 1994b). These spectral lines can be used for electron density determination if the electron density is high $\left(>10^{12} \mathrm{~cm}^{-3}\right)$.

Electron excitation data for the B-like ions was reviewed by Sampson et al. (1994). Extensive datasets are available for this sequence and include those of Sampson et al. (1986) who used their own non-standard CB exchange method, with relativistic corrections. These are generally in very good agreement with the IC DW calculations using the UCL code. These data have now been 
superseded by more recent R-matrix and fully relativistic DW calculations.

\subsubsection{II}

The observed energy levels for the $2 \mathrm{~s}^{2} 2 \mathrm{p}, 2 \mathrm{~s} 2 \mathrm{p}^{2}, 2 \mathrm{p}^{3}$, $2 \mathrm{~s}^{2} 3 \mathrm{~s}$, and $2 \mathrm{~s}^{2} 3 \mathrm{p}$ configurations are from Kelly (1987) and are complete. Oscillator strengths and $A$ values are taken from Dankwort \& Trefftz (1978), Nussbaumer \& Storey (1981), Lennon et al. (1985), and Wiese \& Fuhr (1995). Radiative transition probabilities for C II have been measured in the laboratory by Fang et al. (1993). R-matrix values of the collision strengths among these levels have been provided by Blum \& Pradhan (1992). For the allowed transitions, the collision strengths generally tend to extrapolate to the proper high energy limit derived from the oscillator strength.

\subsubsection{N III}

Observed energies for the 20 fine structure levels of the $2 \mathrm{~s}^{2} 2 \mathrm{p}, 2 \mathrm{~s} 2 \mathrm{p}^{2}, 2 \mathrm{p}^{3}, 2 \mathrm{~s}^{2} 3 \mathrm{~s}, 2 \mathrm{~s}^{2} 3 \mathrm{p}$ and $2 \mathrm{~s}^{2} 3 \mathrm{~d}$ configurations are from Moore (1993) and Kelly (1987). Oscillator strengths and $A$ values have been provided for all of the same levels by Stafford et al. (1993) except for the ground level fine structure transition where the $A$ value is from Nussbaumer \& Storey (1979). Stafford et al. (1994) report R-matrix calculations of Maxwellian-averaged collision strengths among all these configurations.

\subsubsection{O IV, Ne VI, Mg VIII, Al IX, Si X, S XII, Ar XIV, Ca XVI and Fe XXII}

The 125 fine structure levels of the $2 \mathrm{~s}^{2} 2 \mathrm{p}, 2 \mathrm{~s} 2 \mathrm{p}^{2}, 2 \mathrm{p}^{3}$ and $2 l 2 l^{\prime} 3 l^{\prime \prime}$ configurations have been included for these ions. The energy levels are from the NIST database (Martin et al. 1995) except for the $2 \mathrm{~s} 2 \mathrm{p}^{2}{ }^{4} \mathrm{P}$ levels which are from Edlen (1981). Widing (1996) has pointed out that the Edlen values seem to identify several lines of Ar XVI arising from the $2 \mathrm{~s} 2 \mathrm{p}^{2}{ }^{4} \mathrm{P}$ levels. For the $n=2$ configurations, the observed energies are known. For the $2 l 2 l^{\prime} 3 l^{\prime \prime}$ configurations, most of the levels are known for O IV but as $Z$ increases, the number of levels for which there are observed energies become fewer and for Fe XXII only a minority of these levels have been identified. Oscillator strengths and $A$ values for the $n=2$ levels were obtained from the same sources as for C II above with additional data from Bhatia et al. (1986). For the $n=3$ levels, the oscillator strengths from the unpublished calculations of Zhang \& Sampson (1995) were used.

Zhang et al. (1994) have provided R-matrix calculations of Maxwellian averaged collision strengths between the 15 fine structure levels in the $2 \mathrm{~s}^{2} 2 \mathrm{p}, 2 \mathrm{~s} 2 \mathrm{p}^{2}$ and $2 \mathrm{p}^{3}$ configurations. The behavior of the collision strengths at the lowest temperatures $(100-500 \mathrm{~K})$ can often vary so rapidly that they can not be well represented with a 5 point spline. Often these values have been ignored so that the accuracy at the lowest temperature is about only a factor of 2. Collision strengths to the $n=3$ levels (110 fine structure levels) have been calculated by Zhang \& Sampson (1995) using the Coulomb-Born method.

One complication for the boron sequence is a level crossing by the $2 \mathrm{~s} 2 \mathrm{p}^{2}{ }^{2} \mathrm{~S}_{1 / 2}$ and ${ }^{2} \mathrm{P}_{1 / 2}$ levels somewhere between calcium $(Z=20)$ and iron $(Z=26)$. Zhang (1995) has confirmed that their published collision strengths involving these two levels must be exchanged to reflect this. Dankwort \& Trefftz (1978) appear to have simply exchanged labels so that of the two, the ${ }^{2} \mathrm{P}_{1 / 2}$ levels always has the higher energy.

Figure 2 shows an example of the use of the Zhang et al. R-matrix collision strengths for the case of Si X. It has been necessary to truncate the calculated collision strengths to temperatures above $310^{4} \mathrm{~K}$. Since $\mathrm{Si} \mathrm{X}$ is formed near $10^{6} \mathrm{~K}$ in collisional equilibrium, there should be no loss of accuracy for collisionally dominated plasmas. The high temperature limit is determined from the oscillator strength. In general, it would be useful to have the oscillator strength calculated for the same atomic model used for the collisional calculations to compare the $\Upsilon$ values to. The average deviation of the spline fit to the scaled collision strengths is about $0.5 \%$.

Ait-Tahar et al. (1996) have recently carried out a Dirac R-matrix calculation for transitions between levels in the $n=2$ configurations of Fe XXII. For the high energies, they find 10\% agreement with RDW (Zhang \& Sampson 1994) and R-matrix (BP + TCC) (Zhang \& Pradhan 1994, 1995). For low energies they find some discrepancies in the resonance structures which need further investigation. They have published sample plots of $\Omega$ 's and their $\Upsilon$ 's are not yet available.

\subsubsection{Ni XXIV}

The atomic model included the same levels as the O IV - Fe XXII ions above. The values for the observed energy levels were taken from NIST (Martin et al. 1995) and Edlen (1981). For the $n=2$ levels, the oscillator strengths and $A$ values were generally from Dankwort \& Trefftz (1978) except for the $2 \mathrm{~s} 2 \mathrm{p}^{2}{ }^{2} \mathrm{~S}_{1 / 2}$ and ${ }^{2} \mathrm{P}_{1 / 2}$ levels which were from Sampson et al. (1986). The ground term fine structure $A$ value was obtained by extrapolating the values of Bhatia et al. (1986). Oscillator strenghts and $A$ values involving the $n=3$ levels are from Zhang $\&$ Sampson (1995). For the $n=2$ levels of Ni XXIV, the collision strengths were taken from the Coulomb-BornExchange calculations of Sampson et al. (1986). For the $n=3$ levels, the collision strengths are those of Zhang \& Sampson (1995).

\subsection{The carbon isoelectronic sequence}

Lines from ions in the carbon isoelectronic sequence have been studied in spectra from the transition region, corona 

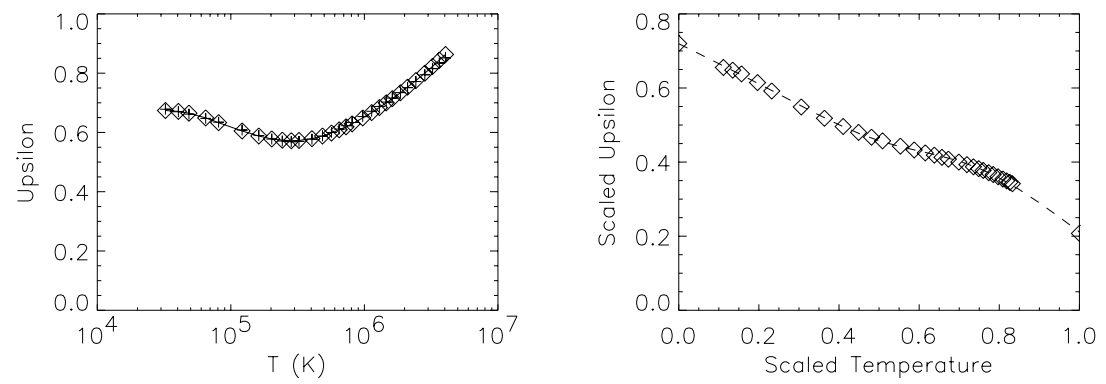

Fig. 2. Collision strengths (Zhang et al. 1994) for Si X $2 \mathrm{~s}^{2} 2 \mathrm{p}{ }^{2} \mathrm{P}_{1 / 2}-2 \mathrm{~s} 2 \mathrm{p}^{2}{ }^{2} \mathrm{D}_{3 / 2}$. Left, the original Maxwellian averaged collision strengths $\Upsilon$, right, the scaled collision strengths with the high energy limit from the Bethe approximation. The scaling follows that of Burgess \& Tully (1992)

and flares. The ground configuration $2 \mathrm{~s}^{2} 2 \mathrm{p}^{2}$ comprises ${ }^{3} \mathrm{P}_{\mathrm{J}},{ }^{1} \mathrm{D}$ and ${ }^{1} \mathrm{~S}$ levels. The relative populations of these levels can be sensitive functions of density. References are given in Mason \& Monsignori Fossi (1994), Dwivedi (1994) and Keenan (1996). Monsignori Fossi \& Landini (1994c) assessed the electron excitation data for the Clike ions. Burgess et al. 1991 have critiqued the R-matrix calculations of Aggarwal (1985b) for Mg VII. They find his treatment for the allowed transitions inadequately accounts for the long range potential. Errors on the order of $15 \%$ could arise and they point out that there may be similar problems with allowed transitions of other ions in this isoelectronic sequence calculated in a similar manner by Aggarwal.

In addition to the density diagnostic ratios for the coronal lines, the solar flare lines from Fe XXI are of particular interest. The UV line at $1354.1 \AA$ has been studied from Skylab and SMM observations and more recently stellar spectra have been obtained with the Goddard High Resolution Spectrograph on the Hubble Space Telescope (Maran et al. 1994). Highly ionized iron lines have been recorded for several stars with EUVE, and electron density values have been deduced for the stellar atmospheres from the Fe XXI line ratios (Dupree et al. 1993).

\subsubsection{N II}

The configurations $2 \mathrm{~s}^{2} 2 \mathrm{p}^{2}, 2 \mathrm{~s} 2 \mathrm{p}^{3}, 2 \mathrm{~s}^{2} 2 \mathrm{p} 3 \mathrm{~s}, 2 \mathrm{~s}^{2} 2 \mathrm{p} 3 \mathrm{p}$, corresponding to 23 fine-structure levels have been included. The experimental energy levels of the NIST database were adopted. The oscillator strengths and $A$ values of Bell et al. (1995) have been adopted. Since Bell's work did not provide radiative transition probabilities for several transitions, a new calculation has been performed using the SSTRUCT package of the University College of London. The new calculation included 17 configurations: $2 \mathrm{~s}^{2} 2 \mathrm{p}^{2}, 2 \mathrm{~s} 2 \mathrm{p}^{3}, 2 \mathrm{p}^{4}, 2 \mathrm{~s}^{2} 2 \mathrm{p} 3 \mathrm{~s}, 2 \mathrm{~s}^{2} 2 \mathrm{p} 3 \mathrm{p}, 2 \mathrm{~s}^{2} 2 \mathrm{p} 3 \mathrm{~d}, 2 \mathrm{~s} 2 \mathrm{p}^{2} 3 \mathrm{~s}$, $2 \mathrm{~s} 2 \mathrm{p}^{2} 3 \mathrm{p}, 2 \mathrm{~s} 2 \mathrm{p}^{2} 3 \mathrm{~d}, 2 \mathrm{~s}^{2} 2 \mathrm{p} 4 \mathrm{~s}, 2 \mathrm{~s}^{2} 2 \mathrm{p} 4 \mathrm{p}, 2 \mathrm{~s}^{2} 2 \mathrm{p} 4 \mathrm{~d}, 2 \mathrm{~s}^{2} 2 \mathrm{p} 4 \mathrm{f}$, $2 \mathrm{~s} 2 \mathrm{p}^{2} 4 \mathrm{~s}, 2 \mathrm{~s} 2 \mathrm{p}^{2} 4 \mathrm{p}, 2 \mathrm{~s} 2 \mathrm{p}^{2} 4 \mathrm{~d}$ and $2 \mathrm{~s} 2 \mathrm{p}^{2} 4 \mathrm{f}$. The resulting radiative transition probabilities have been corrected for the differences between experimental and theoretical values of the transition's wavelengths. The corrected values have been inserted where Bell's values were unavailable.

The effective collision strengths $\Upsilon$ for all transitions are taken from the R-matrix calculations of Stafford et al. (1994). Effective collision strengths are calculated in the electron temperature range $510^{3}$ to $1.2510^{5} \mathrm{~K}$. Lennon \& Burke (1994) have also recently published collision data for the C-sequence, including N II. Some of their values differ from Stafford et al. by more than $50 \%$. The problem is that the position of a large resonance near threshold dramatically effects the low temperature $\Upsilon$ values. The differences between the two sets of calculations must be taken as a measure of the uncertainty in the calculations. The exact position of such resonances is extremely difficult to determine.

\subsubsection{O III}

The adopted atomic model for O III includes 46 fine structure levels of the $2 \mathrm{~s}^{2} 2 \mathrm{p}^{2}, 2 \mathrm{~s} 2 \mathrm{p}^{3}, 2 \mathrm{p}^{4}, 2 \mathrm{~s}^{2} 2 \mathrm{p} 3 \mathrm{~s}, 2 \mathrm{~s}^{2} 2 \mathrm{p} 3 \mathrm{p}$, and $2 \mathrm{~s}^{2} 2 \mathrm{p} 3 \mathrm{~d}$ configurations. Experimental energies for all levels come from the NIST database and have been used to calculate transition wavelengths. Oscillator strengths and $A$ values for all possible transitions between the 46 energy levels are taken from Bhatia \& Kastner (1993a). For transitions between the levels of the ground configuration (first 5 levels) the R-Matrix Maxwellian averaged collision strengths of Lennon \& Burke (1994) were adopted. They report values in the electron temperature range $10^{3}-10^{5} \mathrm{~K}$. Effective collision strengths for optically allowed transitions between the levels belonging to $2 \mathrm{~s}^{2} 2 \mathrm{p}^{2}$ and $2 \mathrm{~s} 2 \mathrm{p}^{3}, 2 \mathrm{p}^{4}$ configurations have been calculated by Aggarwal (1985a) for temperatures between $510^{3}$ and 5 $10^{5} \mathrm{~K}$. Collision strengths for the remaining forbidden and intercombination transitions among these levels are provided by Aggarwal (1983) for temperatures between 2.5 $10^{3}$ and $610^{5} \mathrm{~K}$. Both calculations have been performed using the R-Matrix method. A comparison between the Maxwellian averaged collision strengths from Lennon \& Burke (1994) and Aggarwal (1983) for transitions between the ground configuration levels shows no significant 
differences. Collision strengths for transitions to the $n=3$ levels are taken from the distorted wave calculations of Bhatia \& Kastner (1993a). Collision strengths are reported for 5 values of incident electron energy at 4,6 , 8, 10 and 12 Rydberg.

\subsubsection{Ne V}

The adopted atomic model for $\mathrm{Ne} \mathrm{V}$ includes 6 configurations, $2 \mathrm{~s}^{2} 2 \mathrm{p}^{2}, 2 \mathrm{~s} 2 \mathrm{p}^{3}, 2 \mathrm{~s}^{2} 2 \mathrm{p} 3 \mathrm{~s}, 2 \mathrm{p}^{4}, 2 \mathrm{~s}^{2} 2 \mathrm{p} 3 \mathrm{p}$, and $2 \mathrm{~s}^{2} 2 \mathrm{p} 3 \mathrm{~d}$, corresponding to 46 fine structure energy levels. Experimental energy levels are taken from the NIST database for all the levels, with the exceptions of levels $2 \mathrm{p}^{4}{ }^{1} \mathrm{D}_{2}$ and ${ }^{1} \mathrm{~S}_{0}$, which come from Edlen (1985). $A$ values and oscillator strengths come from Bhatia \& Doschek (1993b), and are provided for all the possible transitions between the 46 energy levels.

Maxwellian averaged collision strengths for transitions between the ground levels (configuration $2 \mathrm{~s}^{2} 2 \mathrm{p}^{2}$ and $2 \mathrm{~s} 2 \mathrm{p}^{3}$ ${ }^{5} \mathrm{~S}_{2}$ ) are taken from Lennon \& Burke (1994). Collision strengths for all other the transitions come from the distorted wave calculations of Bhatia \& Doschek (1993b). Collision strengths are provided for 3 values of incident electron energies at 10, 15 and 20 Rydberg. Collision strengths have also been calculated using the R-Matrix by Aggarwal (1985b, 1986) for allowed transitions and by Aggarwal (1984) for forbidden and intercombination transitions.

\subsubsection{Mg VII, Si IX}

The configurations $2 \mathrm{~s}^{2} 2 \mathrm{p}^{2}, 2 \mathrm{~s} 2 \mathrm{p}^{3}, 2 \mathrm{~s}^{2} 2 \mathrm{p} 3 \mathrm{~s}, 2 \mathrm{p}^{4}, 2 \mathrm{~s}^{2} 2 \mathrm{p} 3 \mathrm{p}$, $2 \mathrm{~s}^{2} 2 \mathrm{p} 3 \mathrm{~d}$, corresponding to 46 fine-structure levels, were included in the atomic models for these 2 ions. Values for the observed energy levels were taken from the NIST database. Transition wavelengths were calculated using observed energy levels, but where these values were not available theoretical energy levels were used. Theoretical energy levels, oscillator strengths, collision strengths and spontaneous transition probabilities for these ions are taken from the calculations of Bhatia \& Doschek (1993c, 1995b) for Si IX and Mg VII respectively. Collision strengths were calculated using the distorted wave approximation for three values of the incident electron energy in the range $12-36$ Rydberg (Mg VII) and 20 - 60 Rydberg (Si IX). For these ions, the incident energies are fairly high above threshold and closely spaced in energy. This presents a problem in extrapolating the collision strengths over the complete energy range from threshold to infinite energy.

R-matrix calculations of allowed transitions in $\mathrm{Mg}$ VII and Si IX have been performed by Aggarwal (1985b, 1986) but may be somewhat inaccurate as discussed by Burgess et al. (1991).

\subsubsection{S XI}

The adopted atomic model for S XI includes the configurations $2 \mathrm{~s}^{2} 2 \mathrm{p}^{2}, 2 \mathrm{~s} 2 \mathrm{p}^{3}, 2 \mathrm{p}^{4}, 2 \mathrm{~s}^{2} 2 \mathrm{p} 3 \mathrm{~s}, 2 \mathrm{~s}^{2} 2 \mathrm{p} 3 \mathrm{p}$ and $2 \mathrm{~s}^{2} 2 \mathrm{p} 3 \mathrm{~d}$, corresponding to 46 fine structure energy levels. Experimental energies were taken from the NIST database while the theoretical energies for all the levels come from Bhatia \& Kastner (1987). Wavelengths have been calculated with the experimental energy levels but where these values were not available the theoretical energies have been used. Radiative transition probabilities and oscillator strengths are reported by Bhatia \& Kastner (1987) for all possible transitions between the levels of the adopted atomic model.

Collision strengths for transitions between all the 46 levels are available from Bhatia \& Kastner (1987). In this paper collision strengths are calculated for only one value of the incident electron energy at 25 Rydberg. The calculation of the Maxwellian-averaged collision strengths is then very arbitrary. For this reason collision strengths for all transitions among the ground levels $\left(2 \mathrm{~s}^{2} 2 \mathrm{p}^{2}\right.$ configuration) and between the ground configuration and the $2 \mathrm{~s} 2 \mathrm{p}^{3}$ configuration have been taken from Mason \& Bhatia (1978). In this paper collision strengths are reported for 3 values of incident electron energy, and the calculation of the Maxwellian-averaged collision strengths is better defined. For the remaining transitions, the effective collision strengths have been calculated using the single energy collision strengths. For these transitions we have artificially introduced the $\Omega(E)$ values at threshold and for $E \rightarrow \infty$ so that they are similar to energy dependence of the same transition for other members of the isoelectronic sequence. A comparison between the effective collision strengths of Mason \& Bhatia (1978) and those of Bhatia \& Kastner (1987) show differences of up to $30 \%$.

\subsubsection{Ar XIII}

The adopted atomic model for Ar XIII includes the configurations $2 \mathrm{~s}^{2} 2 \mathrm{p}^{2}$ and $2 \mathrm{~s} 2 \mathrm{p}^{3}$, corresponding to 15 fine structure energy levels. Experimental energies come from the NIST database and have been used to calculate transition wavelengths. Theoretical energy levels, $A$ values, oscillator strengths and collision strengths for all the possible transitions have been taken from Dere et al. (1979). Collision strengths are calculated using the distorted wave approximation, including the configurations $2 \mathrm{~s}^{2} 2 \mathrm{p}^{2}, 2 \mathrm{~s} 2 \mathrm{p}^{3}$ and $2 \mathrm{p}^{4}$, for 3 values of the incident electron energy at 15,30 and 45 Rydberg.

\section{7. $C a X V$}

The adopted atomic model for Ca XV includes the configurations $2 \mathrm{~s}^{2} 2 \mathrm{p}^{2}$ and $2 \mathrm{~s} 2 \mathrm{p}^{3}$, corresponding to 15 fine structure energy levels. Experimental energies come from the NIST database and have been used to calculate transition 
wavelengths. Oscillator strengths and $A$ values are from the calculations of Froese-Fischer \& Saha (1985).

For the Ca XV $2 \mathrm{~s}^{2} 2 \mathrm{p}^{2}$ and $2 \mathrm{~s} 2 \mathrm{p}^{3}$ configurations, we use the R-matrix collision strengths of Aggarwal et al. (1991a). We have also considered distorted wave calculations for this ion (Bhatia \& Doschek 1993a; Dere et al. 1979). For the transitions within the $n=2$ configurations, it was not possible to obtain satisfactory fits to the Bhatia \& Doschek (1993a) collision strengths because the incident energies are too closely bunched and too high above threshold. In these cases, extrapolation of the collision strength values to threshold and the high energy limit (nondipole) is very uncertain. Dere et al. (1979) published collision strengths for $\mathrm{Ca} \mathrm{XV}$ at lower energies. Although most of their values matched those of Bhatia \& Doschek at $45 \mathrm{Ryd}$, a few transitions differed. The probable reason is that Dere et al. did not carry out the DW calculations to partial wave values as high as Bhatia and Doschek. The Dere et al. values at 45 Ryd may not have fully converged. Consequently, it was not possible to construct a consistent set of distorted wave collision strengths that spanned a sufficient range in energy. In addition, the resonance contribution is significant for the forbidden transitions within the ground configuration. Indeed, for some of these transitions, the $\Upsilon$ 's at $310^{6}$ derived from Bhatia \& Doschek (1993a) differ by more than a factor 2 from Aggarwal's values.

\subsubsection{Fe XXI}

For Fe XXI, 36 fine-structure levels in the configurations $2 \mathrm{~s}^{2} 2 \mathrm{p}^{2}, 2 \mathrm{~s} 2 \mathrm{p}^{3}, 2 \mathrm{~s}^{2} 2 \mathrm{p} 3 \mathrm{~s}, 2 \mathrm{~s}^{2} 2 \mathrm{p} 3 \mathrm{~d}, 2 \mathrm{p}^{4}$ were included. The observed energies are mostly from the NIST database and supplemented by a few from Mason et al. (1979). Oscillator strengths and $A$ values have been computed using the SSTRUCT package. The adopted atomic model includes 17 configurations: $2 \mathrm{~s}^{2} 2 \mathrm{p}^{2}, 2 \mathrm{~s} 2 \mathrm{p}^{3}, 2 \mathrm{p}^{4}, 2 \mathrm{~s}^{2} 2 \mathrm{p} 3 \mathrm{~s}$, $2 \mathrm{~s}^{2} 2 \mathrm{p} 3 \mathrm{p}, 2 \mathrm{~s}^{2} 2 \mathrm{p} 3 \mathrm{~d}, 2 \mathrm{~s} 2 \mathrm{p}^{2} 3 \mathrm{~s}, 2 \mathrm{~s} 2 \mathrm{p}^{2} 3 \mathrm{p}, 2 \mathrm{~s} 2 \mathrm{p}^{2} 3 \mathrm{~d}, 2 \mathrm{~s}^{2} 2 \mathrm{p} 4 \mathrm{~s}$, $2 \mathrm{~s}^{2} 2 \mathrm{p} 4 \mathrm{p}, 2 \mathrm{~s}^{2} 2 \mathrm{p} 4 \mathrm{~d}, 2 \mathrm{~s}^{2} 2 \mathrm{p} 4 \mathrm{f}, 2 \mathrm{~s} 2 \mathrm{p}^{2} 4 \mathrm{~s}, 2 \mathrm{~s} 2 \mathrm{p}^{2} 4 \mathrm{p}, 2 \mathrm{~s} 2 \mathrm{p}^{2} 4 \mathrm{~d}$ and $2 \mathrm{~s} 2 \mathrm{p}^{2} 4 \mathrm{f}$. The calculated radiative transition probabilities have been corrected for the differences between experimental and theoretical values of each transition's wavelength.

Collision strengths from Aggarwal (1991) have been adopted for transitions involving the $2 \mathrm{~s}^{2} 2 \mathrm{p}^{2}, 2 \mathrm{~s} 2 \mathrm{p}^{3} 2 \mathrm{p}^{4}$ configurations. They have been calculated using the closecoupling method for 20 values of electron energy in the range 20-300 Rydberg. Collision strengths for transitions from the ground configuration to $2 \mathrm{~s}^{2} 2 \mathrm{p} 3 \mathrm{~s}$ and $2 \mathrm{~s}^{2} 2 \mathrm{p} 3 \mathrm{~d}$ configuration come from Mason et al. (1979). They are calculated with the distorted wave method for 3 values of electron energy in the range $20-100$ Rydberg. The energies specified for the incident electron cover a sufficiently large range, at least for the $n=2$ excitations, that the Maxwellian integral is well determined. For several transitions, a comparison with the R-matrix calculations of
Aggarwal (1991) was made and generally the agreement was found to be quite good. It is noted that electron collision rates to the $n=4$ levels have recently been published by Phillips et al. (1996). These will be included in future releases of CHIANTI.

\subsection{The nitrogen isoelectronic sequence}

The nitrogen isoelectronic sequence provides some interesting diagnostics for coronal ions, cf. Bhatia \& Mason (1980a) and recent work by Dwivedi, Mohan and colleagues referenced in Dwivedi (1994). Kato (1994) has reviewed the existing data for the nitrogen isoelectronic sequence. She found that for several important ions of this sequence very few data are available (Ne IV to Mn XIX), while O II and Fe XX have been studied quite extensively. Most of the existing calculations have been carried out using the distorted wave approximation.

\subsubsection{O II}

The adopted atomic model for O II includes the configurations $2 \mathrm{~s}^{2} 2 \mathrm{p}^{3}, 2 \mathrm{~s} 2 \mathrm{p}^{4}$ and $2 \mathrm{p}^{5}$, corresponding to 15 fine-structure energy levels. The experimental energies are taken from the NIST database. These energies were used to calculate the transition wavelengths. Since no experimental energy values were available for the configuration $2 \mathrm{p}^{5}$, wavelengths for transitions originated from the $2 \mathrm{p}^{5}$ levels were computed using theoretical energies.

Maxwellian averaged collision strengths are taken from McLaughlin \& Bell (1994). Effective collision strengths are provided for all transitions between 11 LS levels (corresponding to configurations $2 \mathrm{~s}^{2} 2 \mathrm{p}^{3}, 2 \mathrm{~s} 2 \mathrm{p}^{4}$, and $2 \mathrm{~s}^{2} 2 \mathrm{p}^{2} 3 \mathrm{~s}$ ). The collisional data were calculated using the R-Matrix method and fitted with a Chebyshev polynomial expansion and tabulated. Effective collision strengths among the fine structure levels have been obtained from the reported LS coupling values by scaling according to the statistical weights of the fine-structure levels involved in each transition. Levels in the $2 \mathrm{~s}^{2} 2 \mathrm{p}^{2} 3 \mathrm{~s}$ configuration have not been included since radiative data for the $2 \mathrm{~s}^{2} 2 \mathrm{p}^{2} 3 \mathrm{~s}$ levels were not available. These levels will be included in future releases of CHIANTI.

For transitions involving the $2 \mathrm{p}^{5}$ configurations and for transitions between the levels of each multiplet, collision strengths have been taken from the unpublished distorted wave calculations Bhatia (1996), provided for 3 values of incident electron energy at 4, 8 and 12 Rydberg. Since collision strengths for all other configurations are provided, a comparison has been carried out between these data and those of McLaughlin \& Bell (1994). The two sets of data show significant differences both in the allowed and in the forbidden transitions. 


\subsubsection{Ne IV}

The adopted atomic model for Ne IV includes 3 configurations: $2 \mathrm{~s}^{2} 2 \mathrm{p}^{3}, 2 \mathrm{~s} 2 \mathrm{p}^{4}$ and $2 \mathrm{p}^{5}$, corresponding to 15 fine-structure energy levels. The experimental energies are taken from the NIST database and are used to calculate the wavelengths.

The radiative data and collision strengths have been taken from unpublished distorted wave calculations of Bhatia (1996). Collision strengths are calculated using the distorted wave method for 3 values of electron energy at 5, 10 and 15 Rydberg.

\subsubsection{Mg VI, Si VIII, S X, Ar XII, Ca XIV}

The atomic model includes two configurations: $2 \mathrm{~s}^{2} 2 \mathrm{p}^{3}$ and $2 \mathrm{~s} 2 \mathrm{p}^{4}$, corresponding to 13 fine-structure levels. Energy levels and transition wavelengths have been calculated using the experimental energy levels of the NIST database.

Radiative transition probabilities and collision strengths for all transitions within $2 \mathrm{~s}^{2} 2 \mathrm{p}^{3}$ and between the $2 \mathrm{~s}^{2} 2 \mathrm{p}^{3}$ and $2 \mathrm{~s} 2 \mathrm{p}^{4}$ configurations are included and are from the calculations of Bhatia \& Mason (1980a). The scattering calculations have been carried out using the configurations $2 \mathrm{~s}^{2} 2 \mathrm{p}^{3}, 2 \mathrm{~s} 2 \mathrm{p}^{4}$ and $2 \mathrm{p}^{5}$, with the distorted wave method, and collision strengths are computed for 3 values of the electron energy between 10 and 45 Rydberg.

\subsubsection{Fe XX}

The configurations included in the atomic model are $2 \mathrm{~s}^{2} 2 \mathrm{p}^{3}$ and $2 \mathrm{~s} 2 \mathrm{p}^{4}$, corresponding to 13 fine-structure energy levels. The relevant atomic data for Fe XX are taken from Bhatia \& Mason (1980b). Both theoretical and experimental energy levels are provided by the authors. Transition wavelengths have been calculated using the experimental values. Collision strengths have been calculated using the distorted wave method for 3 values of electron energy: 20, 50 and 100 Rydberg.

\subsection{The oxygen isoelectronic sequence}

Spectroscopic diagnostics for the oxygen-like transition region and coronal ions were explored by Raju \& Dwivedi (1978). The solar flare lines from Fe XIX have been studied in XUV (Loulergue et al. 1985) and X-ray (Bhatia et al. 1989) spectra.

The oxygen isoelectronic sequence has not been treated extensively in the literature. The only close-coupling calculations are those of Butler \& Zeippen (1994) who have calculated thermal-averaged collision strengths for transitions between the levels within the ground configuration of ions from F II to Ar XI, for electron temperatures ranging from $10^{3}$ to $10^{5} \mathrm{~K}$. Distorted wave calculations have been performed for all the most abundant ions, including transitions between the ground configuration and excited ones. A comparison between distorted wave and close-coupling collision strengths for the lighter ions shows a generally acceptable agreement. Only S IX shows some differences between the two different methods, where the close coupling data sometimes show greater thermal-averaged collision strengths at low temperatures. Since the maximum abundance temperature for oxygen-like ions with $Z \geq 16$ is $\geq 10^{6} \mathrm{~K}$ we have adopted distorted wave calculations also for the transitions in the ground configuration for $\mathrm{Si}$ VII and above.

The case of Ne III and $\mathrm{Mg} \mathrm{V}$ is different. The electron temperature for the maximum abundance for these ions falls in the range $10^{5}-10^{5.5} \mathrm{~K}$ and it is important to include the close-coupling effective collision strengths since the effects of resonances are important.

The main deficiency of the adopted distorted wave collision strengths is that they have been calculated only for three values of the incident electron energy near threshold. Consequently, the behavior of the non-dipole collisions strengths at high energy is not well determined.

\subsubsection{Ne III and $\mathrm{Mg} \mathrm{V}$}

For Ne III and Mg V, the 10 fine-structure levels belonging to the $2 \mathrm{~s}^{2} 2 \mathrm{p}^{4}, 2 \mathrm{~s} 2 \mathrm{p}^{5}$ and $2 \mathrm{p}^{6}$ configurations have been included. Observed energy levels are taken from the NIST database and they are used to calculate the transition wavelengths. The radiative data are from the unpublished calculations of Bhatia (1996) which also provide collision strengths for all the transitions between these 10 levels. Distorted wave collision strengths for Ne III are computed for three values of the incident electron energy in the range $5-15$ Rydberg, and for Mg V, 4 values of electron energy are available, in the range $10-30$ Rydberg.

Butler \& Zeippen (1994) report close-coupling effective collision strengths for the ground configuration transitions. These effective collision strengths are provided for 11 values of the electron temperature ranging from $10^{3}$ to $10^{5} \mathrm{~K}$. This temperature range is insufficient for an accurate extrapolation to high energy. The collision strengths provided by Bhatia do allow a good determination of the effective collision strengths in the temperature range $10^{6}-510^{6} \mathrm{~K}$. Thus the two different sets of data have been merged and the resulting effective collision strengths have been inserted in the CHIANTI database. No significant inconsistencies between the two different sets of data were found.

Bhatia's collision strengths have been adopted for all other transitions.

\subsubsection{Si VII, S IX, Ar XI}

The atomic model includes the $2 \mathrm{~s}^{2} 2 \mathrm{p}^{4}, 2 \mathrm{~s} 2 \mathrm{p}^{5}$ and $2 \mathrm{p}^{6}$ configurations, corresponding to 10 fine structure energy levels.

Experimental energy levels from the NIST database have been adopted. These values have been used to 
calculate all the transition wavelengths. The experimental energy of the $2 \mathrm{p}^{6}{ }^{1} \mathrm{~S}_{0}$ level of Ar XI is unknown and all the wavelengths of the transitions involving this level have been computed using theoretical energies. Theoretical energy levels, radiative transition probabilities and collision strengths for Si VII, S IX and Ar XI have been provided by Bhatia et al. (1979).

The scattering problem has been solved using the distorted wave approximation. Collision strengths have been calculated for 3 values of electron energy: 10, 15, 20 Rydberg for Si VII and S IX, and 15, 20, 25 Rydberg for Ar XI. Again, this is a fairly restricted range in energy for determining the energy dependence of the collision strengths.

\subsubsection{Ca XIII}

The atomic model of Ca XIII includes 4 configurations: $2 \mathrm{p}^{4}, 2 \mathrm{~s} 2 \mathrm{p}^{5}, 2 \mathrm{p}^{6}$ and $2 \mathrm{p}^{3} 3 \mathrm{~s}$, corresponding to 20 finestructure energy levels. The experimental energies of the NIST database have been adopted, but the values for the levels $2 \mathrm{p}^{3} 3 \mathrm{~s}{ }^{5} \mathrm{~S}_{2}$ and ${ }^{3} \mathrm{P}_{0,1,2}$ are unknown. Transition wavelengths have been calculated using the experimental energy levels, but if these values were not available, the theoretical ones have been used. The theoretical energy levels, radiative transition probabilities and collision strengths are taken from the unpublished calculations of Bhatia (1996).

The collision strengths were computed using the distorted wave approximation. They have been calculated for 5 values of the electron energy in the range between 40 and 60 Rydberg. The collisional data are available for all transition between and within the listed configurations. These data agree well with Mason (1975) for the $n=2$ transitions.

Baliyan \& Bhatia (1994) published R-matrix collision data for Ca XIII with a more extensive set of target configurations. Unfortunately, their results are in LS coupling and they do not tabulate the $\Upsilon$ 's, so we have not used them in the CHIANTI database. However, they have carried out a very careful comparison of the R-matrix and distorted wave results, giving particular attention to the partial wave convergence. In general, they found good agreement (better than 30\%) with the Bhatia distorted wave results, although they suggest that the target wavefunctions could be improved. They also found the resonance structure to be significant for low energies.

\subsubsection{Fe XIX}

The adopted atomic model includes $2 \mathrm{~s}^{2} 2 \mathrm{p}^{4}, 2 \mathrm{~s} 2 \mathrm{p} 5$ and $2 \mathrm{p}^{6}$ configurations, corresponding to 10 fine-structure energy levels. Theoretical energy levels, radiative transition probabilities and collision strengths come from Loulergue et al. (1985), who also provide the experimental energy levels.
The latter energies are used to calculate the transition wavelengths.

The authors show that for calculating the radiative transition probabilities, there is no significant improvement in extending this basis to higher configurations than those listed above. The collision strengths are calculated using the distorted wave method for 3 values of incident electron energy, 22.5, 45 and 90 Rydberg.

\subsection{The fluorine isoelectronic sequence}

\subsubsection{Ne II, Mg IV, Si VI, S VIII and Ca XII}

For the fluorine sequence ions Ne II through Ca XII, only the two lowest configurations having a total of 3 fine structure levels have been included: $2 \mathrm{~s}^{2} 2 \mathrm{p}^{5}{ }^{2} \mathrm{P}_{3 / 2,1 / 2}$ and $2 \mathrm{~s} 2 \mathrm{p}^{6}{ }^{2} \mathrm{~S}_{1 / 2}$. The experimental energy levels are from Kelly (1987) for Ne II and from the NIST database (Martin et al. 1995) for the others. $A$ values between the ground configuration levels are from Martin et al. (1995) and from Blackford \& Hibbert (1994) for the others. Collision strengths between the 2 ground configuration levels are from the calculations of Saraph \& Tully (1994). Sources for collision strength to the $2 \mathrm{~s} 2 \mathrm{p}^{6}$ level are: Ne II (Bhatia 1996, unpublished), Mg IV (Mohan et al. 1988), Si VI (Mohan \& Le Dourneuf 1990), S VIII (Mohan et al. 1987) and Ca XII (Mason 1975).

\subsubsection{Fe XVIII and Ni XX}

The 113 fine structure levels of the $2 \mathrm{~s}^{2} 2 \mathrm{p}^{5}, 2 \mathrm{~s} 2 \mathrm{p}^{6}, 2 \mathrm{~s}^{2} 2 \mathrm{p}^{4} 3 l$, $2 \mathrm{~s} 2 \mathrm{p}^{5} 3 l$ and $2 \mathrm{p}^{6} 3 l$ configurations are included. Observed energy levels have been taken from the NIST database (Martin et al. 1995). The primary source of oscillator strengths and $A$ values were the calculations of Sampson et al. (1991). Additional radiative rates were taken from Blackford \& Hibbert (1994) and Cornille et al. (1992). Sampson et al. (1991) have calculated relativistic distorted-wave collision strengths for ions with $Z \geq 22$ and have been incorporated into the CHIANTI database. These calculations are generally in good agreement with the distorted wave calculations of Cornille et al. (1992) for Fe XVIII.

\subsection{The neon isoelectronic sequence}

\subsubsection{Si V}

The adopted atomic model includes 4 configurations: $2 \mathrm{~s}^{2} 2 \mathrm{p}^{6}, 2 \mathrm{~s}^{2} 2 \mathrm{p}^{5} 3 \mathrm{~s}, 2 \mathrm{~s}^{2} 2 \mathrm{p}^{5} 3 \mathrm{p}$ and $2 \mathrm{~s}^{2} 2 \mathrm{p}^{5} 3 \mathrm{~d}$, corresponding to 27 fine-structure levels. The experimental energy levels are taken from the NIST database and are used to calculate all the transition wavelengths. Radiative transition probabilities and collision strengths come from the calculations of Bhatia et al. (1985). Radiative data are available for all transition between the listed configurations. 
Collision strengths were calculated using the distorted wave method for only one value of the incident electron energy, 15 Rydberg. For dipole transitions, a second energy point is available in the Bethe approximation high energy limit so that the integration over a Maxwellian velocity distribution can be performed. For the nondipole transitions, the variation of the collision strength with energy has been estimated from the same transition in Fe XVII, either by setting the collision strength to be constant with energy or to go to zero at high energies.

\subsubsection{S VII}

The adopted atomic model for S VII includes four configurations: $2 \mathrm{~s}^{2} 2 \mathrm{p}^{6}, 2 \mathrm{~s}^{2} 2 \mathrm{p}^{5} 3 \mathrm{~s}, 2 \mathrm{~s}^{2} 2 \mathrm{p}^{5} 3 \mathrm{p}$ and $2 \mathrm{~s}^{2} 2 \mathrm{p}^{5} 3 \mathrm{~d}$ corresponding to 27 fine-structure levels. The adopted experimental energy levels are taken from the NIST database and they are used to calculate all the transition wavelengths. The theoretical energy levels, $A$ values and oscillator strengths are taken from Hibbert et al. (1993). Radiative data are calculated for transitions to the ground configuration, between $2 \mathrm{~s}^{2} 2 \mathrm{p}^{5} 3 \mathrm{~s}$ and $2 \mathrm{~s}^{2} 2 \mathrm{p}^{5} 3 \mathrm{p}$, between $2 \mathrm{~s}^{2} 2 \mathrm{p}^{5} 3 \mathrm{p}$ and $2 \mathrm{~s}^{2} 2 \mathrm{p}^{5} 3 \mathrm{~d}$. $A$ values for the forbidden transitions $2 \mathrm{~s}^{2} 2 \mathrm{p}^{6}{ }^{1} \mathrm{~S}_{0}-2 \mathrm{~s}^{2} 2 \mathrm{p}^{5} 3 \mathrm{~s}^{3} \mathrm{P}_{2}$ and $2 \mathrm{~s}^{2} 2 \mathrm{p}^{5} 3 \mathrm{~s}^{3} \mathrm{P}_{1}-{ }^{3} \mathrm{P}_{0}$ have been extrapolated from the corresponding values of the other ions of the neon isoelectronic sequence.

Thermal-averaged collision strengths are taken from Mohan et al. (1990). The collision strengths are calculated using the close coupling method for all transitions from the ground level to $2 \mathrm{~s}^{2} 2 \mathrm{p}^{5} 3 \mathrm{~s}, 2 \mathrm{~s}^{2} 2 \mathrm{p}^{5} 3 \mathrm{p}$ and $2 \mathrm{~s}^{2} 2 \mathrm{p}^{5} 3 \mathrm{~d}$ configurations. The data are tabulated for 20 values of electron temperature ranging from $510^{4}$ to $10^{6} \mathrm{~K}$. These data are calculated in LS coupling. Since the ground level consists of a single level, fine structure thermal-averaged collision strengths for transitions involving multiplets have been distributed according to their statistical weights.

\subsubsection{Ar IX, Ca XI, Ni XIX}

The included configurations are $2 \mathrm{~s}^{2} 2 \mathrm{p}^{5} \mathrm{nl}$ and $2 \mathrm{~s} 2 \mathrm{p}^{6} \mathrm{nl}$ with $n=3,4$ and $l=0,1,2,3$. This atomic model includes 89 fine structure levels. The experimental energy levels are taken from the NIST database. These values have been used to calculate transition wavelengths, but where experimental energy levels were lacking (Ca XI and Ni XIX), theoretical values of Zhang et al. (1987) have been used.

Zhang et al. (1987) also calculated radiative transition probabilities for electric dipole transitions between the ground level and the excited levels. These rates provide decay rates for only a few (14) of the upper levels. Additional oscillator strengths and $A$ values have been taken from Hibbert et al. (1993). Nevertheless for the majority of energy levels belonging to the higher energy configurations $2 \mathrm{~s}^{2} 2 \mathrm{p}^{5} 4 l$ and $2 \mathrm{~s} 2 \mathrm{p}^{6} n l$, radiative data are not available. Consequently, it is not possible to construct a model of the statistical level populations that include these levels and so they have been omitted from the adopted atomic model. Since these levels have very high energy and they are not metastable we do not expect any significant change in the level populations and line intensities in the synthetic spectra of these ions. The adopted atomic model includes all levels of configurations $2 \mathrm{~s}^{2} 2 \mathrm{p}^{6}, 2 \mathrm{~s}^{2} 2 \mathrm{p}^{5} 3 \mathrm{~s}, 2 \mathrm{~s}^{2} 2 \mathrm{p}^{5} 3 \mathrm{p}$ and $2 \mathrm{~s}^{2} 2 \mathrm{p}^{5} 3 \mathrm{~d}$, and the levels $2 \mathrm{~s} 2 \mathrm{p}^{6} 3 \mathrm{p}^{3} \mathrm{P}_{1},{ }^{1} \mathrm{P}_{1}, 2 \mathrm{~s}^{2} 2 \mathrm{p}^{5} 4 \mathrm{~s}^{3} \mathrm{P}_{1}$ and ${ }^{1} \mathrm{P}_{1}, 2 \mathrm{~s}^{2} 2 \mathrm{p}^{5} 4 \mathrm{~d}{ }^{3} \mathrm{P}_{1},{ }^{3} \mathrm{D}_{1},{ }^{1} \mathrm{P}_{1}, 2 \mathrm{~s} 2 \mathrm{p}^{6} 4 \mathrm{p}{ }^{3} \mathrm{P}_{1}$ and ${ }^{1} \mathrm{P}_{1}$. The total number of energy levels is 36 .

Collisional data are taken from Zhang et al. (1987) who employed a Coulomb-Born-exchange method. The collision strengths are calculated for 9 values of the incident electron energy in threshold units in the range $1-15$, allowing a straightforward determination of the collision strength values from threshold to the high energy limit. Collisional data are available for all transitions connecting the ground level and the 88 excited levels. Collision strengths for transitions involving the omitted levels have not been inserted in the database, though they are available in CHIANTI format.

\subsubsection{Fe XVII}

The adopted atomic model includes seven configurations: $2 \mathrm{~s}^{2} 2 \mathrm{p}^{6}, 2 \mathrm{~s}^{2} 2 \mathrm{p}^{5} 3 \mathrm{~s}, 2 \mathrm{~s}^{2} 2 \mathrm{p}^{5} 3 \mathrm{p}, 2 \mathrm{~s}^{2} 2 \mathrm{p}^{5} 3 \mathrm{~d}, 2 \mathrm{~s} 2 \mathrm{p}^{6} 3 \mathrm{~s}, 2 \mathrm{~s} 2 \mathrm{p}^{6} 3 \mathrm{p}$, $2 \mathrm{~s} 2 \mathrm{p}^{6} 3 \mathrm{~d}$ corresponding to 37 fine structure energy levels. The experimental energy levels for the lowest 26 levels are from Bhatia \& Doschek (1992) and the rest are from the NIST database. It should be noted that Bhatia \& Doschek preserve the term designations of the lower $Z$ members of the sequence. The experimental energies have been used to calculate the transition wavelengths. Where no experimental energy values were available, theoretical ones have been used. Theoretical energy values, $A$ values, oscillator strengths and collision strengths are provided by Bhatia \& Doschek (1992) for all transitions between the 37 levels of the adopted atomic model. Distorted wave collision strengths are calculated at five incident electron energies in the range $77-254$ Rydberg. These collision strengths can be compared those from a similar calculations by Cornille et al. (1994).

\subsection{The sodium isoelectronic sequence: $\mathrm{Mg} I I, \mathrm{Al} I I I, \mathrm{Si}$ IV, S VI, Ar VIII, Ca X, Fe XVI, Ni XVIII}

The 21 fine structure levels of the $3 l, 4 l$, and $5 l$ configurations have been included. The observed energy levels are from the NIST database (Martin et al. 1995). Oscillator strengths and $A$ values are from Sampson et al. (1990) except for the $4 \mathrm{f}-5 \mathrm{~g}$ transitions where the hydrogenic values of Wiese et al. (1966) are used.

Relativistic distorted wave collision strengths have been provided by Sampson et al. (1990) over a wide energy range and are used in the CHIANTI database. Cornille et al. (1997) have calculated distorted wave results for Fe XVI $(n=3,4$ and 5$)$. They discuss the 
importance of high partial wave contributions, even for the electric quadrupole transitions. Their values agree well with those of Sampson et al. (1990). Tayal (1994) published R-matrix values for the $n=3,4$ configurations. He included the resonance contribution, which is particularly important at lower temperatures $\left(<10^{6} \mathrm{~K}\right)$. New Rmatrix calculations are in progress by Eissner et al. (1996) as part of the Iron Project. They find that some collision rates published by Tayal (1994) are very sensitive to the position of large resonances near threshold.

\subsection{The magnesium isoelectronic sequence}

\subsubsection{Si III}

The adopted atomic model for Si III includes the configurations $3 \mathrm{~s}^{2}, 3 \mathrm{~s} 3 \mathrm{p}, 3 \mathrm{p}^{2}, 3 \mathrm{~s} 3 \mathrm{~d}, 3 \mathrm{~s} 4 \mathrm{~s}$ and $3 \mathrm{~s} 4 \mathrm{p}$, corresponding to 20 fine structure energy levels. The experimental energy levels are taken from the NIST database and have been used to calculate the transition wavelengths. The theoretical energy levels come from Baluja \& Hibbert (1980). Oscillator strengths and $A$ values come from Dufton et al. (1983a). Since only a few transitions are treated in that paper, we have been performed new calculations for all the possible transition in the adopted atomic model. The calculation has been carried out with SSTRUCT, including the configurations $3 \mathrm{~s}^{2}, 3 \mathrm{~s} 3 \mathrm{p}, 3 \mathrm{p}^{2}, 3 \mathrm{~s} 3 \mathrm{~d}, 3 \mathrm{~s} 4 \mathrm{~s}, 3 \mathrm{~s} 4 \mathrm{p}, 3 \mathrm{~s} 4 \mathrm{~d}$, $3 \mathrm{~s} 4 \mathrm{f}, 3 \mathrm{p} 3 \mathrm{~d}, 3 \mathrm{~d}^{2}, 3 \mathrm{p} 4 \mathrm{~s}, 3 \mathrm{p} 4 \mathrm{p}, 3 \mathrm{p} 4 \mathrm{~d}$ and $3 \mathrm{p} 4 \mathrm{f}$. The resulting $A$ values and oscillator strengths have been adopted for all the transition for which values were not provided by Dufton et al. (1983a). A comparison between SSTRUCT results and Dufton et al. (1983a) radiative transition probabilities shows no significant differences.

Adopted collision data come from Dufton \& Kingston (1989). They provide coefficients for least-squares polynomial fits for thermal averaged collision strengths between the 20 lowest states (corresponding to the 20 fine-structure levels adopted by CHIANTI). Thermal averaged collision strengths have been calculated with the R-matrix program for the temperature interval between $10^{3.8}$ and $10^{5.2} \mathrm{~K}$, for which the Si III ionization fraction is significant. Fine structure transitions between triplets were reported, while transitions between singlets and triplets are presented in LS coupling. The authors show that the values for fine structure transitions between singlets and triplets may be obtained by scaling the collisional data by the appropriate degeneracies which we have done here.

\subsubsection{S V, Ar VII, Ca IX, Ni XVII}

The adopted atomic model for S V, Ar VII, Ca IX and Ni XVII includes the configurations $3 \mathrm{~s}^{2}, 3 \mathrm{~s} 3 \mathrm{p}, 3 \mathrm{p}^{2}, 3 \mathrm{~s} 3 \mathrm{~d}$ and $3 \mathrm{~s} 4 \mathrm{~s}$, corresponding to 16 fine-structure levels. The experimental energy levels are taken the NIST database. Experimental energy levels have been used to calculate the transition wavelengths. Where no experimental values were available, the theoretical ones have been used.
Theoretical energy levels, $A$ values, oscillator strengths and collisional data are taken from Christensen et al. (1986). Radiative transition probabilities are available for electric dipole transitions between $3 \mathrm{~s}^{2}$ and $3 \mathrm{~s} 3 \mathrm{p}$ configurations, and between $3 \mathrm{~s} 3 \mathrm{p}$ and $3 \mathrm{p}^{2}, 3 \mathrm{~s} 3 \mathrm{~d}$ and $3 \mathrm{~s} 4 \mathrm{~s}$ configurations. Collision strengths are calculated with the distorted wave method for three incident electron energies for all possible transition between the 16 fine-structure levels of the adopted atomic model.

\subsubsection{Fe XV}

The atomic model for Fe XV includes configurations $3 \mathrm{~s}^{2}$, 3s $3 \mathrm{p}, 3 \mathrm{p}^{2}$, 3s3d, 3p3d, 3s4s, 3s4p, 3s4d, 3p4s, 3p4d, 3p4f. The radiative data and electron scattering data are taken from Bhatia et al. (1997). The collision strengths, calculated in the distorted wave approximation, are given for 3 energies, 25, 50 and 75 Rydbergs. Fits were made from the levels in the $3 \mathrm{~s}^{2}$ and $3 \mathrm{~s} 3 \mathrm{p}$ configurations up to all the other configurations. One limitation of these calculations was the omission of the $3 \mathrm{~d}^{2}$ configuration. New calculations for the $n=3$ configurations have just been completed (Bhatia $\&$ Mason 1997). These are in good agreement with the earlier distorted wave results by Christensen et al. (1985). Dufton et al. (1990) compared their R-matrix results with Christensen et al. and also found good agreement (better than 20\%). They did not publish their collision data and it is not available. Eissner et al. (1996) are carrying out new R-matrix calculations for Fe XV as part of the Iron Project. They find that, contrary to Dufton et al. 's conclusions, the resonance structure is important for some of the Fe XV transitions and could make a significant difference to the excitation rates.

\subsection{The aluminum isoelectronic sequence}

\subsubsection{Si II}

The atomic model for Si II includes five configurations, $3 \mathrm{~s}^{2} 3 \mathrm{p}, 3 \mathrm{~s} 3 \mathrm{p}^{2}, 3 \mathrm{~s}^{2} 3 \mathrm{~d}, 3 \mathrm{~s}^{2} 4 \mathrm{~s}$ and $3 \mathrm{~s}^{2} 4 \mathrm{p}$, corresponding to 15 fine structure levels. The experimental energy levels are taken from the NIST database and are used for calculating the transition wavelengths. Experimental energies are available for all levels. Electric dipole allowed transitions are taken from Lanzafame (1994). Radiative transition probabilities for forbidden and intercombination transitions are obtained with SSTRUCT. The following configurations have been included in the calculation: $3 \mathrm{~s}^{2} 3 \mathrm{p}, 3 \mathrm{~s} 3 \mathrm{p}^{2}, 3 \mathrm{~s}^{2} 3 \mathrm{~d}, 3 \mathrm{~s}^{2} 4 \mathrm{~s}, 3 \mathrm{~s}^{2} 4 \mathrm{p}, 3 \mathrm{~s}^{2} 4 \mathrm{~d}, 3 \mathrm{~s}^{2} 4 \mathrm{f}, 3 \mathrm{p}^{3}$, 3s3p3d, 3s 3d $\mathrm{d}^{2}, 3 \mathrm{p} 3 \mathrm{~d}^{2}, 3 \mathrm{~d}^{3}, 3 \mathrm{p}^{2} 3 \mathrm{~d}, 3 \mathrm{~s} 3 \mathrm{p} 4 \mathrm{~s}, 3 \mathrm{~s} 3 \mathrm{p} 4 \mathrm{p}, 3 \mathrm{~s} 3 \mathrm{p} 4 \mathrm{~d}$ and $3 \mathrm{~s} 3 \mathrm{p} 4 \mathrm{f}$. These radiative transition probabilities have been calculated using the theoretical energy levels but have been corrected to take into account the experimental energies.

Thermal averaged collision strengths are taken from the R-matrix calculations of Dufton \& Kingston (1991a). Thermal averaged collision strengths are tabulated for 6 
values of the electron temperature ranging from $10^{3.6}$ to $10^{4.6} \mathrm{~K}$.

\subsubsection{S IV}

The adopted atomic model for S IV includes the 3 configurations $3 \mathrm{~s}^{2} 3 \mathrm{p}, 3 \mathrm{~s} 3 \mathrm{p}^{2}$ and $3 \mathrm{~s}^{2} 3 \mathrm{~d}$, corresponding to 12 finestructure energy levels. Experimental energies were available from the NIST database for all the included levels, and have been used to calculate transition wavelengths. Oscillator strengths and $A$ values are mainly taken from Bhatia et al. (1980), who provide values for transitions between all the levels of the adopted atomic model. Radiative decay rates for the intercombination lines between levels $3 \mathrm{~s}^{2} 3 \mathrm{p}{ }^{2} \mathrm{P}$ and $3 \mathrm{~s} 3 \mathrm{p}^{2}{ }^{4} \mathrm{P}$ have been taken from Dufton et al. (1982).

Collisional data come from 3 sources. For transitions between $3 \mathrm{~s}^{2} 3 \mathrm{p}{ }^{2} \mathrm{P}$ and $3 \mathrm{~s} 3 \mathrm{p}^{2}{ }^{4} \mathrm{P}$, collision strengths have been taken from the R-matrix calculations of Dufton et al. (1982). They present Maxwellian-averaged collision strengths in the electron temperature range $10^{4}$ to $10^{5.6} \mathrm{~K}$. A comparison between these results and the close-coupling calculations of Bhadra \& Henry (1980) and the distorted wave values of Bhatia et al. (1980) has shown that the Dufton values are larger than the others at low temperatures, due to scattering resonances. The main difference between Dufton's calculations and Bhadra's and Bhatia's is that the former explicitly delineates the resonances in the collision strengths. Collision strengths for transitions from $3 \mathrm{~s}^{2} 3 \mathrm{p}{ }^{2} \mathrm{P}$ and $3 \mathrm{~s} 3 \mathrm{p}^{2}{ }^{4} \mathrm{P}$ to the remaining levels of the $3 \mathrm{~s} 3 \mathrm{p}^{2}$ configuration come from Bhadra \& Henry (1980). They provide fine structure collision strengths for five values of the incident electron energy in the range 1.3 to 6 Rydberg, calculated with the closecoupling method. For transitions involving the $3 \mathrm{~s}^{2} 3 \mathrm{~d}$ configuration, the Bhatia et al. (1980) collision strengths have been adopted. They are calculated with the distorted wave method for three values of incident electron energy in the range 2 to 6 Rydberg.

\subsubsection{Fe XIV}

The model for Fe XIV includes 12 fine structure levels of the $3 \mathrm{~s}^{2} 3 \mathrm{p}, 3 \mathrm{~s} 3 \mathrm{p}^{2}$ and $3 \mathrm{~s}^{2} 3 \mathrm{~d}$ configurations. The observed energy levels are from the NIST database. Transition probabilities are taken from Froese Fischer \& Liu (1986).

The R-matrix calculations of Dufton \& Kingston (1991b) give upsilons for all transitions from the ground $3 \mathrm{~s}^{2} 3 \mathrm{p}$ configuration to the $3 \mathrm{~s} 3 \mathrm{p}^{2}$ and $3 \mathrm{~s}^{2} 3 \mathrm{~d}$ configurations and are used here. No significant differences were found between this work, the earlier work by Mason (1975) and the more recent work of Bhatia \& Kastner (1993b). Storey et al. (1996) give upsilons for the ground ${ }^{2} \mathrm{P}_{1 / 2}-{ }^{2} \mathrm{P}_{3 / 2}$ transition calculated using the R-matrix codes. Only the upsilons above $10^{4} \mathrm{~K}$ were fitted as the simple 5 point spline could not be applied accurately to the complete set of upsilons.

Although the Storey et al. upsilons were around $50 \%$ greater than the Dufton \& Kingston upsilons, this had little effect on the level balance as the dominant process populating the ${ }^{2} \mathrm{P}_{3 / 2}$ level is cascades from upper levels.

\subsection{The silicon isoelectronic sequence}

\subsubsection{Fe XIII}

The model of the Fe XIII ion includes 27 fine structure levels belonging to the $3 \mathrm{~s}^{2} 3 \mathrm{p}^{2}, 3 \mathrm{~s} 3 \mathrm{p}^{3}$ and $3 \mathrm{~s}^{2} 3 \mathrm{p} 3 \mathrm{~d}$ configurations. The observed energy levels are from the NIST database. A 24 configuration model of Fe XIII was used in SSTRUCT to generate transition probabilities. Most importantly, transition probabilities were derived to depopulate the metastable $3 \mathrm{~s}^{2} 3 \mathrm{p} 3 \mathrm{~d}^{3} \mathrm{~F}_{4}$ level, which accounts for some $20 \%$ of the level balance at densities of around $10^{12}-10^{13} \mathrm{~cm}^{-3}$. In the work of Brickhouse et al. (1995), this level was omitted as there existed no data in the literature to de-populate the level, and explains the discrepancies between the CHIANTI results and theirs.

The distorted wave calculations of Fawcett \& Mason (1989) were used in favor of the more recent R-matrix calculations of Tayal (1995), principally because the Rmatrix results differed for some transitions (e.g., $3-13$, $4-14$ ) by a factor of 2 , the reasons for which were not adequately explained. Also, upsilons were not published by Tayal for transitions up to the $3 \mathrm{~s}^{2} 3 \mathrm{p} 3 \mathrm{~d}$ configuration or for the $3 \mathrm{~s} 3 \mathrm{p}^{3}{ }^{5} \mathrm{~S}_{2}$ level. It is to be noted that Fawcett \& Mason (1989) provide only collision strengths for transitions out of the ground configuration and so the effects of electron collisional excitation out of the metastable ${ }^{3} \mathrm{~F}_{4}$ level have not been included.

\subsubsection{Ni XV}

As with Fe XIII, the model of the Ni XV ion includes 27 fine structure levels belonging to the $3 \mathrm{~s}^{2} 3 \mathrm{p}^{2}, 3 \mathrm{~s} 3 \mathrm{p}^{3}$ and $3 \mathrm{~s}^{2} 3 \mathrm{p} 3 \mathrm{~d}$ configurations. Experimental energies were available for only 17 of the 27 levels and so theoretical values were used for the remaining levels.

Accurate transition probabilities were calculated using SSTRUCT with a 35 configuration model. Again, we find that magnetic quadrupole transitions are significant in helping to depopulate the metastable $3 \mathrm{~s} 3 \mathrm{p}^{2} 3 \mathrm{~d}^{3} \mathrm{~F}_{4}$ level. We also note that there are no collision strengths available that might allow this level to be de-populated by electron collisional processes to configurations other than $3 \mathrm{~s}^{2} 3 \mathrm{p}^{2}$, which may affect the level population significantly.

The distorted wave calculations of Mason (1996b) are used to provide collision strengths at one energy (10 Ryd) for all of the $3 \mathrm{~s}-3 \mathrm{p}$ and $3 \mathrm{p}-3 \mathrm{~d}$ transitions. Four configurations were included in these calculations $-3 \mathrm{~s}^{2} 3 \mathrm{p}^{2}$, $3 \mathrm{~s} 3 \mathrm{p}^{3}, 3 \mathrm{~s} 3 \mathrm{p}^{2} 3 \mathrm{~d}$ and $3 \mathrm{p}^{4}$-but collision strengths were not calculated for transitions to or from $3 \mathrm{p}^{4}$. For dipole 
transitions, the single collision strength and the value of the high energy limit derived from the oscillator strength define the energy dependence of the collision strength. For other transitions, the collision strength is assumed to be constant with energy.

\subsection{The phosphorus isoelectronic sequence}

Forbidden lines of S II such as $\lambda 6717$ and $\lambda 6731$ are observed in planetary nebulae and their intensity ratio provides a good indicator of electron density for values near $10^{3} \mathrm{~cm}^{-3}$ (Osterbrock 1989). Fe XII line ratios are sensitive diagnostics of electron density for coronal conditions (Dere 1982).

\subsubsection{S II}

For S II, 28 fine structure levels of the $3 \mathrm{~s}^{2} 3 \mathrm{p}^{3}, 3 \mathrm{~s} 3 \mathrm{p}^{4}$, $3 \mathrm{~s}^{2} 3 \mathrm{p}^{2} 3 \mathrm{~d}$, and $3 \mathrm{~s}^{2} 3 \mathrm{p}^{2} 4 \mathrm{~s}$ configurations have been included. Energy levels based on observed spectra are from the NIST database (Martin et al. 1995). Sources for oscillator strengths and $A$ values for S II include Mendoza \& Zeippen (1982), Ho \& Henry (1983), Huang (1984), Fawcett (1986). There are large differences among these various sources for the values of the radiative constants. In addition, none provide a radiative decay rate for the $3 \mathrm{~s}^{2} 3 \mathrm{p}^{2} 3 \mathrm{~d}^{4} \mathrm{~F}_{9 / 2}$ level. Consequently, we have used a single set of allowed and forbidden radiative rates for $\mathrm{S}$ II calculated with the SSTRUCT program (Binello 1996). The effective collision rates have been calculated by Cai \& Pradhan (1993) using the R-matrix program.

We note that new R-matrix calculations have recently been carried out for S II by Ramsbottom et al. (1996), including 43 fine-structure levels, up to the $n=4$ configurations. These will be assessed in the next release of CHIANTI.

\subsubsection{Fe XII}

The model of the Fe XII ion includes 41 fine structure levels belong to the $3 \mathrm{~s}^{2} 3 \mathrm{p}^{3}, 3 \mathrm{~s} 3 \mathrm{p}^{4}$ and $3 \mathrm{~s}^{2} 3 \mathrm{p}^{2} 3 \mathrm{~d}$ configurations. Energy levels are from the NIST database and Jupen et al. (1994). Transition probabilities were taken from a recent SSTRUCT run performed by Binello (1996) using a 24 configuration model for Fe XII.

No advances over the distorted wave calculations of Flower (1977) have been made for the important EUV transitions of Fe XII. Tayal et al. (1987) presented Rmatrix upsilons for the transitions within the ground $3 \mathrm{~s}^{2}$ $3 \mathrm{p}^{3}$ configuration, which we use to supplement the Flower data. Tayal \& Henry (1988) published R-matrix collision data between the $3 \mathrm{~s}^{2} 3 \mathrm{p}^{2}$ and $3 \mathrm{~s} 3 \mathrm{p}^{3}$ configurations. Their collision strengths agree with Flower's values to within $30 \%$. Some strange effects in the Tayal et al. and Tayal \& Henry data arise through the use of pseudo-resonances (see Mason 1994). New calculations of electron excitation data for Fe XII as part of the Iron Project (Binello et al.
1996). We await these results for incorporation into the CHIANTI database.

We note that the most important requirements for this ion are collision strengths to the $3 \mathrm{~s}^{2} 3 \mathrm{p}^{2} 3 \mathrm{~d}$ configuration and out of the metastable levels in that configuration, which can have substantial populations even at densities of $10^{8}-10^{10} \mathrm{~cm}^{-3}$.

\subsection{The sulphur isoelectronic sequence: Fe XI}

The model for Fe XI includes 47 fine structure levels of the $3 \mathrm{~s}^{2} 3 \mathrm{p}^{4}, 3 \mathrm{~s} 3 \mathrm{p}^{5}, 3 \mathrm{~s}^{2} 3 \mathrm{p}^{3} 3 \mathrm{~d}$ and $3 \mathrm{p}^{6}$ configurations. Most of the energy levels were taken from the NIST database and a few from the work of Jupen et al. (1994). Observed energies for many levels do not exist. A 13 configuration model of Fe XI was used to derive transition probabilities with SSTRUCT. It was found that adding the extra configurations significantly altered the mixing coefficients of the $3 \mathrm{~s}^{2} 3 \mathrm{p}^{3} 3 \mathrm{~d}{ }^{3} \mathrm{~S}_{1},{ }^{3} \mathrm{P}_{1}$ and ${ }^{1} \mathrm{P}_{1}$ levels, thus affecting their oscillator strengths.

New distorted wave calculations by Bhatia \& Doschek (1996) have finally superseded the work of Mason (1975). Collision strengths at 3 energies were calculated. In view of the changes in mixing coefficients just noted and because there were significant differences between SSTRUCT and Bhatia \& Doschek for other transitions, we decided to rescale all the $3 \mathrm{~s}^{2} 3 \mathrm{p}^{4}-3 \mathrm{~s}^{2} 3 \mathrm{p}^{3} 3 \mathrm{~d}$ collision strengths by the ratio of SSTRUCT oscillator strengths to the Bhatia \& Doschek oscillator strengths. One consequence of this was to increase the strength of the ${ }^{3} \mathrm{P}_{2}-{ }^{1} \mathrm{P}_{1}$ transition, and so we identify this as the $188.30 \AA$ line, rather than the ${ }^{3} \mathrm{P}_{2}-{ }^{3} \mathrm{~S}_{1}$ identification of Jupén et al. (1994).

\subsection{The chlorine isoelectronic sequence: Fe $X$}

The model for $\mathrm{Fe} \mathrm{X}$ includes 54 fine structure levels in the $3 s^{2} 3 p^{5}, 3 s 3 p^{6}, 3 s^{2} 3 p^{4} 3 d$ and $3 s 3 p^{5} 3 d$ configurations. Energy levels are from the NIST database and from Jupen et al. (1994). Experimental energies of many of the $3 \mathrm{~s} 3 \mathrm{p}^{5} 3 \mathrm{~d}$ levels are still unknown. Oscillator strengths together with all transition probabilities were derived using a 12 configuration model in SSTRUCT. The configurations used were $3 \mathrm{~s}^{2} 3 \mathrm{p}^{5}$, $3 \mathrm{~s} 3 \mathrm{p}^{6}, 3 \mathrm{~s}^{2} 3 \mathrm{p}^{4} 3 \mathrm{~d}, 3 \mathrm{~s} 3 \mathrm{p}^{5} 3 \mathrm{~d}, 3 \mathrm{~s}^{2} 3 \mathrm{p}^{3} 3 \mathrm{~d}^{2}, 3 \mathrm{~s}^{2} 3 \mathrm{p}^{4} 4 \mathrm{~s}, 3 \mathrm{~s}^{2} 3 \mathrm{p}^{4} 4 \mathrm{p}$, $3 \mathrm{~s}^{2} 3 \mathrm{p}^{4} 4 \mathrm{~d}, 3 \mathrm{~s}^{2} 3 \mathrm{p}^{4} 4 \mathrm{f}, 3 \mathrm{~s} 3 \mathrm{p}^{4} 3 \mathrm{~d}^{2}$ and $3 \mathrm{p}^{6} 3 \mathrm{~d}$. The transition probabilities were found to be consistent with those of Fawcett (1991) to within 5\%.

Bhatia \& Doschek (1995a) presented new distorted wave calculations for $\mathrm{Fe} \mathrm{X}$ that were an improvement over the results of Mason (1975); however, for the $3 \mathrm{~s}^{2} 3 \mathrm{p}^{5}$ ${ }^{2} \mathrm{P}-3 \mathrm{~s} 3 \mathrm{p}^{6}{ }^{2} \mathrm{~S}$ transitions, the collision strengths were severely under-estimated and so, for these transitions only, the accurate SSTRUCT oscillator strengths were used to scale upwards the collision strengths. As part of the Iron Project, Pelan \& Berrington (1995) calculated upsilons for the ground ${ }^{2} \mathrm{P}_{3 / 2}-{ }^{2} \mathrm{P}_{1 / 2}$ transition using the $\mathrm{R}$-matrix 
program. The inclusion of resonance structure was found to increase the excitation rate by a factor of 10 for low temperatures, having significant consequences on the level balance of the ion, and so we use the Pelan \& Berrington upsilons for this transition. It is to be noted that Rmatrix calculations have been performed for Fe X by Mohan et al. (1994); however question marks have been raised over this work by Pelan \& Berrington (1995) and Foster et al. (1996) and so we retain the distorted wave calculations for the present.

\subsection{The argon isoelectronic sequence: Fe IX}

For Fe IX, the 13 levels of the $3 \mathrm{p}^{6}$ and $3 \mathrm{p}^{5} 3 \mathrm{~d}$ configurations were included. Energy levels from the NIST database were used. A 10 configuration model of Fe IX was used in SSTRUCT to generate transition probabilities. The distorted wave calculations of Fawcett \& Mason (1991) were used to fit and scale the collision strengths among these levels. Although collisional data was also given for the $3 \mathrm{p}^{5} 4 \mathrm{~s}$ configuration, we note that this would lead to two extra metastable levels $\left({ }^{3} \mathrm{P}_{0}\right.$ and $\left.{ }^{3} \mathrm{P}_{2}\right)$. However, it is evident that the $3 \mathrm{~s} 3 \mathrm{p}^{5} 3 \mathrm{~d}$ and $3 \mathrm{~s} 3 \mathrm{p}^{4} 3 \mathrm{~d}^{2}$ configurations are energetically lower than $3 \mathrm{p}^{5} 4 \mathrm{~s}$, and so the two levels would actually decay via these configurations. The apparent metastable nature of the two levels is thus purely a consequence of the neglect of the $3 \mathrm{~s} 3 \mathrm{p}^{5} 3 \mathrm{~d}$ and $3 \mathrm{~s} 3 \mathrm{p}^{4} 3 \mathrm{~d}^{2}$ configurations in the level balance, and so we ignore the $3 \mathrm{p}^{5} 4 \mathrm{~s}$ configuration in this work. Our $g f$ value for the ${ }^{1} \mathrm{~S}-{ }^{1} \mathrm{P}$ transition (which gives rise to the strong line at $171.07 \AA$ ) was found to be $25 \%$ lower than that given by Fawcett \& Mason and so their collision strengths, for this transition only, were scaled proportionately.

\subsection{The potassium isoelectronic sequence}

\subsubsection{Ca II}

We note that Burgess et al. (1996) have published and fitted non-exchange DW electron excitation data for Ca II using OMEUPS. These will be included in the next release of CHIANTI.

\subsubsection{Fe VIII}

The present model of Fe VIII includes the $3 \mathrm{p}^{6} 3 \mathrm{~d}, 3 \mathrm{p}^{6} 4 \mathrm{p}$, $3 \mathrm{p}^{6} 4 \mathrm{f}, 3 \mathrm{p}^{6} 5 \mathrm{f}, 3 \mathrm{p}^{6} 6 \mathrm{f}, 3 \mathrm{p}^{6} 7 \mathrm{f}$ configurations. Observed energies for the 12 fine structure levels are from the NIST database. Oscillator strengths and $A$ values are from Czyzak \& Krueger (1966), Fawcett (1989) and NIST. Czyzak \& Krueger (1966) have calculated a number of collision strengths for Fe VIII using the Coulomb-Born approximation. Pindzola et al. (1989) have calculated both close-coupling and distorted wave cross sections for the $3 \mathrm{~d}-4 \mathrm{~s}$ and $3 \mathrm{~d}-4 \mathrm{p}$ and $4 \mathrm{~s}-4 \mathrm{p}$. Their close-coupling and distorted wave cross sections tend to show good agreement. The values of Pindzola et al. collision strengths for the $3 \mathrm{~d}-4 \mathrm{p}$ are about a factor of $3-4$ lower than the values calculated by Czyzak \& Krueger. The collision rates of Pindzola for the $3 \mathrm{~d}-4 \mathrm{p}$ transition have been used and the data of Czyzak \& Krueger for the others. Calculations of excitation rates to the $3 \mathrm{p}^{6} 3 \mathrm{~d}^{2}$ configuration are not available and this is a major weakness of the model. In fact, the present values of the collision strengths appear to be of limited accuracy.

\subsection{The calcium isoelectronic sequence: Fe VII}

The 9 fine structure levels of the ground term $3 \mathrm{p}^{6} 3 \mathrm{~d}^{2}$ have been included. Energy levels are taken from the NIST database (Martin et al. 1995) and the oscillator strengths and $A$ values are those of Nussbaumer \& Storey (1982). All of the lines involve forbidden transitions and the shortest wavelength is $1490 \AA$. Collision strengths among the 9 levels of the ground term $3 \mathrm{p}^{6} 3 \mathrm{~d}^{2}$ have been calculated by Norrington \& Grant (1987) and their averages over a Maxwellian velocity distribution tabulated by Keenan \& Norrington (1987). Their values are in good agreement with the distorted wave values of Nussbaumer \& Storey (1982) but diverge at higher energies. The values of Keenan \& Norrington (1987) have been included in the CHIANTI data base. Lines from this ion provide potentially useful density diagnostics for electron densities between $10^{5}$ and $10^{8} \mathrm{~cm}^{-3}$.

\subsection{The manganese isoelectronic sequence: Fe II}

Fe II is a complex ion and produces a large number of lines that are observed at infrared, visible and ultraviolet wavelengths in a variety of astrophysical sources. It plays a major role in radiative losses from solar and stellar chromospheres (Anderson \& Athay 1989).

The model of Fe II uses 142 levels of the quartet and sextet terms of the $3 \mathrm{~d}^{6} 4 \mathrm{~s}, 3 \mathrm{~d}^{7}$ and $3 \mathrm{~d}^{6} 4 \mathrm{p}$ configurations. New calculations of dipole transition probabilities have been made by Nahar (1995). These calculations used the energy levels of Fe II from Johansson (1978) as well as unpublished values by Johansson to arrive at a fairly complete description of the energy levels of Fe II. $A$ values for forbidden transitions are from the NIST compilation (Martin et al. 1995). Recently, Zhang \& Pradhan (1995) have calculated collisions strengths among the 142 fine structure levels levels and are all included in the CHIANTI database. At densities characteristic of stellar chromospheres, the lowest 23 levels can have significant populations.

\section{Observed and predicted spectra: 50 to $1100 \AA$}

The goal of the CHIANTI project was to develop, to the greatest extent possible, the most complete and accurate database for reproducing astrophysical spectra. In order to determine how well these goals have been met, we have 
collected lists of spectral lines and their wavelengths observed in the solar spectrum between 50 and $1100 \AA$. At this stage, we know that the CHIANTI database is incomplete below $50 \AA$, largely because of the omission of the hydrogen and helium isoelectronic sequences. Beyond $1100 \AA$, lines of neutral species, which are not included in CHIANTI at this point, begin to dominate the spectrum. Observed spectra of the non-flaring Sun include Behring et al. (1972, 1976), Burton \& Ridgely (1970), Feldman \& Doschek (1991), Freeman \& Jones (1970), Thomas \& Neupert (1994), and Widing \& Sandlin (1968). The list of Widing and Sandlin has been expanded by Widing (1996) to include unidentified lines not reported in the original paper. Spectra of the flaring Sun include Acton et al. (1985), Dere (1978) and Kastner et al. (1974). The line list of Dere (1978) has been updated to include the additions of Dufton et al. (1983b), Feldman et al. (1985), Jupen (1984), Keenan et al. (1992), Widing (1982) and Widing et al. (1986).

Table 3 lists observed wavelengths, CHIANTI wavelengths, transitions, predicted intensity information and the references for observations of each line. The first column provides an average value of the wavelengths observed in the various reported spectra. Reported wavelengths that differed by less than a chosen wavelength difference $\Delta \lambda$ were generally simply averaged to arrive at the observed wavelength quoted in Table 3 . For wavelengths below $400 \AA, \Delta \lambda$ was set to $0.03 \AA$, for wavelengths between 400 and $600 \AA, \Delta \lambda$ was set to $0.04 \AA$ and for wavelengths above $600 \AA$, $\Delta \lambda$ was set to $0.05 \AA$. The second column contains the wavelength in the CHIANTI data base. In those cases were the transition is not in the CHIANTI data base, a wavelength is reported and the denoted by a intensity value of... in the intensity column. Most of these wavelengths are from Kelly (1987). The third column shows the ion giving rise to the spectral line and the fourth column provides the transition. The fourth column gives the intensity expected from a solar flare using a differential emission measure distribution derived by Dere \& Cook (1979). The intensities are calculated from the differential emission measure, which spans the temperature range $310^{4}$ to $2.510^{7} \mathrm{~K}$, with the elemental abundances of Allen (1973) and the ionization equilibria of Arnaud \& Rothenflug (1985). The intensity values should only be used as a guide to the identification and the problem of blending and should be used in a relative sense. Lines with predicted intensities less than 10 were not considered likely to be observed. Some identifications should be considered simply coincidental, for example high-temperature lines seen in quiet Sun spectra of Behring et al. Predicted spectral lines with intensities above 200 were included even if there was no reported line at that wavelength. These cases are noted by a ... in Col. 1. Only lines for which there are accurate values of the energy levels available based on observed spectra are included. The last column provides the reference where the observation was reported in the literature.

Table 3 indicates that the CHIANTI data base provides a very nearly complete reproduction of astrophysical emission line spectra between 50 and $1100 \AA$, at least in terms of observed spectral lines. Many new identifications for previously unknown lines have been found but many lines remain unidentified. The Table also provides information on the likelihood of problems with blending. Based on our experience in putting the observational data together for this Table, the observations between 170 and $600 \AA$ appear to be the most consistent. At shorter wavelengths, lines are often reported by one observer but not another. At longer wavelengths, the observations do not appear to have been made with sufficient sensitivity. Observations with the SUMER instrument on SOHO should help correct the deficiencies in the long wavelength portion of Table 3 .

Future papers will describe the comparison between observed spectral line intensities and prediction by CHIANTI. Preliminary work has already been performed on this comparison in order to assure the basic correctness of the CHIANTI data base. For example, EUVE spectra of Procyon have been compared with the spectra computed with the CHIANTI database for a quiet sun differential emission measure and EUVE spectra of Au Mic in a flaring state have been compared with the CHIANTI database for a solar flare differential emission measure. In addition, the SERTS spectra have also been compared with predictions from the CHIANTI database. Detailed comparison with these spectra are in progress but our work to date assures us of the reliability of the CHIANTI database.

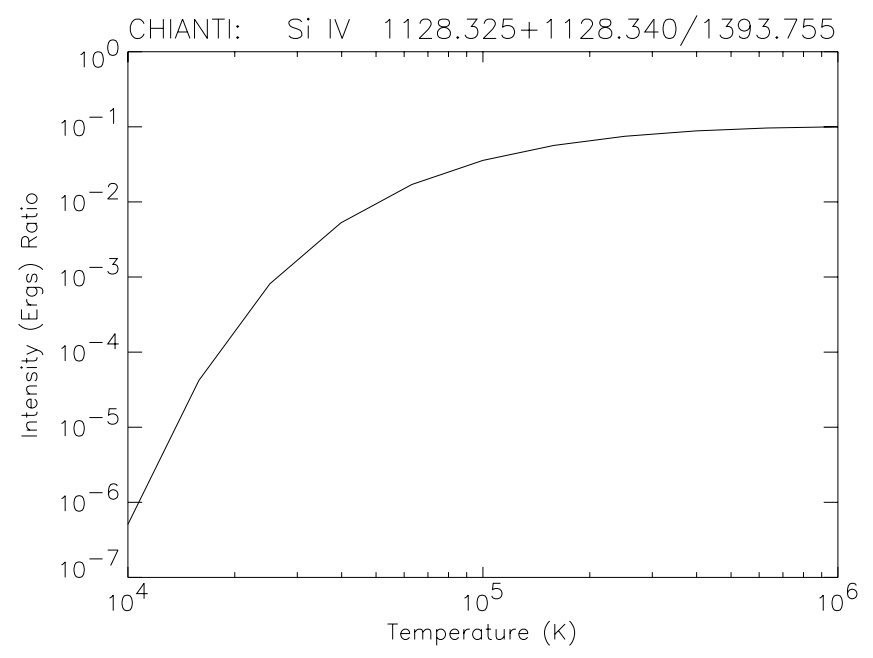

Fig. 3. Temperature-sensitive line ratio of Si IV calculated from the CHIANTI database 
CHIANTI: $\quad$ Si IX $349.794+349.873 / 341.949$

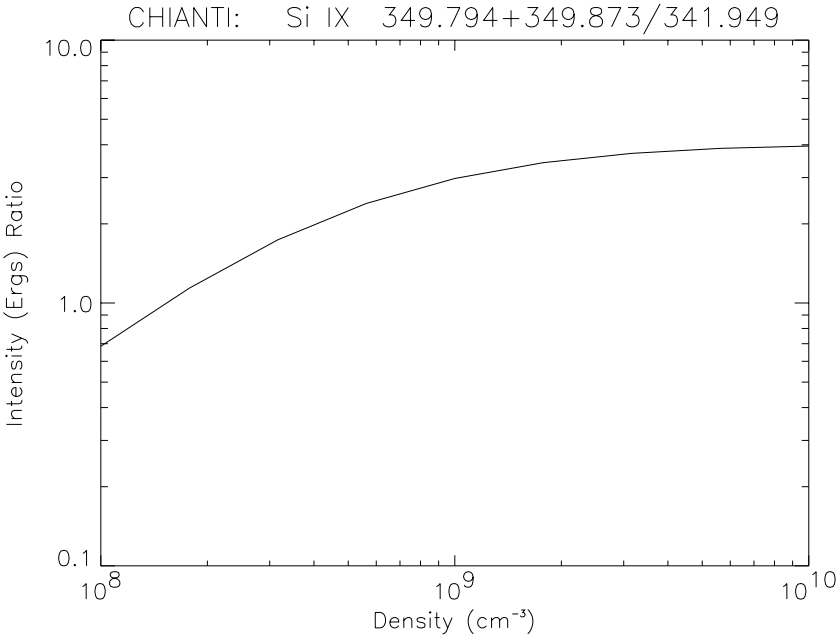

Fig. 4. Density-sensitive line ratio of Si IX calculated from the CHIANTI database

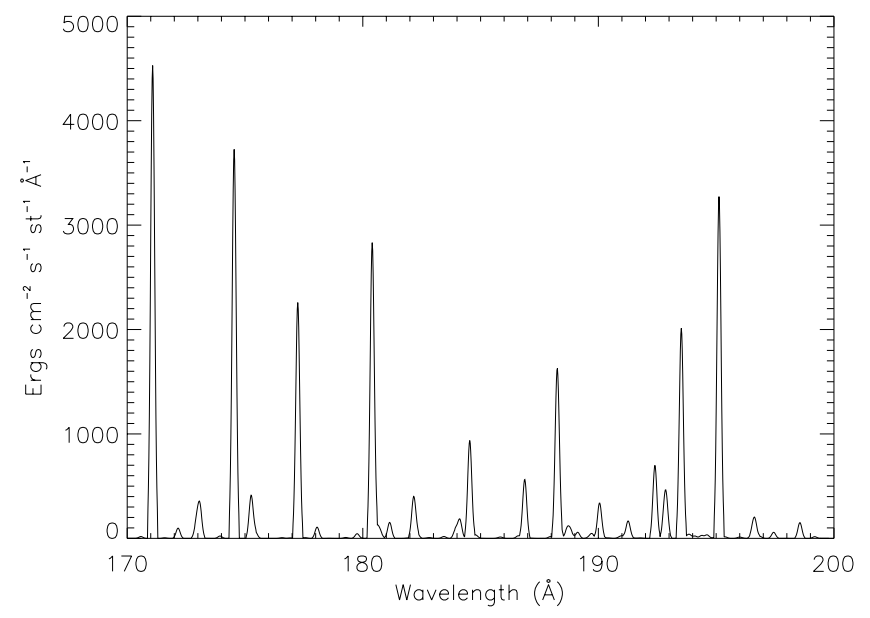

Fig. 5. Synthetic spectrum of the quiet Sun between 160 and $200 \AA$

\section{Illustrative calculations with the CHIANTI database}

The CHIANTI database is quite flexible and can be easily adapted for use in a number of situations. Its uses include the calculation of temperature- and density-sensitive line ratios such as shown in Figs. 3 and 4. The numerator in the Si IX line ratio in Fig. 4 sums the contributions of two lines that would be blended by spectrographs with resolving powers that are currently available. Blending is a common problem and can be readily assessed with the CHIANTI database.

Another intended use of the CHIANTI database is for computations of synthetic spectra for comparison with observed spectra, regardless of spectral resolution. Again, blending can be an important aspect of these studies. A synthetic spectrum of a quiet solar region at wavelengths between 160 and $200 \AA$ is shown in Fig. 5, for a FWHM spectral resolution of $0.2 \AA$.

\section{Availability of the CHIANTI data base}

The CHIANTI database and the corresponding set of IDL programs are freely available from the anonymous FTP server louis14.nrl.navy.mil in the pub/chianti directory. A README file in that directory explains the procedures for copying the Unix compressed tar files to a user's computer. The IDL routines assume a Unix file structure but, with relatively minor modifications, they could be adapted to other operating systems.

\section{Brunella Monsignori Fossi}

Sadly, during the course of this work, our co-author and colleague, Brunella Monsignori Fossi died suddenly and prematurely. Her enthusiasm, support and friendship is a great loss not only to us but to the whole of the solar and stellar physics communities.

Acknowledgements. The success of this database has been dependent on a number of researchers who have made their data available to us in electronic form, often in advance of publication. These include W. Martin and A. Musgrove (NIST), A. Bhatia (GSFC), H. Zhang and A. Pradhan (O.S.U.), R. Sampson (PSU), K.G. Widing (NRL), K.A. Berrington, J. Pelan, and C. Ramsbottom (QUB). KPD acknowledges financial support for this project from the Ultraviolet, Visible and Gravitational Astrophysics program of NASA. HEM and PRY acknowledge the financial support of PPARC. We would also like to thank the referee, Dr. Rolf Mewe, for his careful reading of the manuscript.

\section{References}

Acton L.W., Bruner M.E., Brown W.A., Fawcett B.C., Schweizer W., Speer R.J., 1985, ApJ 291, 865

Aggarwal K.M., 1983, ApJS 52, 387

Aggarwal K.M., 1984, ApJS 54, 1

Aggarwal K.M., 1985a, A\&A 146, 149

Aggarwal K.M., 1985b, ApJS 58, 289

Aggarwal K.M., 1986, ApJS 61, 699

Aggarwal K.M., 1991, ApJS 77, 677

Aggarwal K.M., Berrington K.A., Keenan F.K., 1991a, ApJS 77,441

Aggarwal K.M., Berrington K.A., Kingston A.E., Pathak A., 1991b, J. Phys. B 24, 1757

Aggarwal K.M., Callaway J., Kingston A.E., Unnikrishnan K., 1992, ApJS 80, 473

Ait-Tahar S., Grant I.P., Norrington P.H., 1996, Phys. Rev. (in press)

Allen C.W., 1973, Astrophysical Quantities. The Athlone Press, London, p. 31

Anderson L.S., Athay R.G., 1989, ApJ 346, 1010

Arnaud M., Rothenflug R., 1985, A\&AS 60, 425

Baliyan K.S., Bhatia A.K., 1994, J. Phys. B 27, 4281

Baluja K.L., Hibbert A., 1980, J. Phys. B 13, L327

Behring W.E., Cohen L., Feldman U., 1972, ApJ 175, 493 
Behring W.E., Cohen L., Feldman U., Doschek G.A., 1976, ApJ 203, 521

Bell K.L., Hibbert A., Stafford R.P., 1995, Phys. Scripta 52, 240

Bely-Dubau F., 1994, Adv. Sp. Res. 14, 15

Berrington K.A., 1994, ADNDT 57, 71

Berrington K.A., Burke P.G., LeDourneuf M., Robb W.D., Taylor K.T., Vo Ky Lan, 1978, Comp. Phys. Comm. 14, 367

Berrington K.A., Burke P.G., Dufton P.L., Kingston A.E., 1985, ADNDT 33, 195

Berrington K.A., Burke V.M., Burke P.G., Scialla S., 1989, J. Phys. B 22, 665

Berrington K.A., Kingston A.E., 1990, unpublished calculations

Bhadra K., Henry R.J.W., 1980, ApJ 240, 368

Bhatia A.K., 1996, unpublished DW calculations

Bhatia A.K., Doschek G.A., 1992, ADNDT 52, 1

Bhatia A.K., Doschek G.A., 1993a, ADNDT 53, 195

Bhatia A.K., Doschek G.A., 1993b, ADNDT 55, 315

Bhatia A.K., Doschek G.A., 1993c, ADNDT 55, 281

Bhatia A.K., Doschek G.A., 1995a, ADNDT 60, 97

Bhatia A.K., Doschek G.A., 1995b, ADNDT 60, 145

Bhatia A.K., Doschek G.A., 1996 (in preparation)

Bhatia A.K., Kastner S.O., 1987, J. Quant. Spect. Rad. Transfer 37,527

Bhatia A.K., Kastner S.O., 1992, ApJS 79, 139

Bhatia A.K., Kastner S.O., 1993a, ADNDT 54, 133

Bhatia A.K., Kastner S.O., 1993b, J. Quant. Spect. Rad. Transfer 49, 609

Bhatia A.K., Mason H.E., 1980a, MNRAS 190, 925

Bhatia A.K., Mason H.E., 1980b, A\&A 83, 380

Bhatia A.K., Mason H.E., 1981, A\&A 103, 324

Bhatia A.K., Mason H.E., 1983, A\&AS 52, 115

Bhatia A.K., Mason H.E., 1986, A\&A 155, 413

Bhatia A.K., Mason H.E., 1997, ADNDT 66, 119

Bhatia A.K., Doschek G.A., Feldman U., 1980, A\&A 86, 32

Bhatia A.K., Feldman U., Doschek G.A., 1979, A\&A 80, 22

Bhatia A.K., Feldman U., Seely J.F., 1985, ADNDT 32, 435

Bhatia A.K., Feldman U., Seely J.F., 1986, ADNDT 35, 319

Bhatia A.K., Fawcett B.C., Lemen J.R., Mason H.E., Phillips K.J.H., 1989, MNRAS 240, 421

Bhatia A.K., Mason H.E., Blancard, C., 1997, ADNDT 66, 83

Binello A.M., 1996, unpublished calculations

Binello A.M., Mason H.E., Storey P.J., Kohl J.L., 1996, unpublished calculations

Blackford H.M.S., Hibbert A., 1994, ADNDT 58, 101

Blum R.D., Pradhan A.K., 1992, ApJS 80, 425

Brickhouse N.S., Raymond J.C., Smith B.W., 1995, ApJS 97, 551

Burgess A., Chidichimo M.C., Tully J.A., 1996, A\&A 300, 627

Burgess A., Chidichimo M.C., Tully J.A., 1997, A\&AS 121, 187

Burgess A., Hummer D.G., Tully J.A., 1970, Phil. Trans. Roy. Soc. Lond. A 266, 225

Burgess A., Shoerey V.B., 1974, J. Phys. B 7, 2403

Burgess A., Tully J.A. 1992, A\&A 254, 436

Burgess A., Mason H.E., Tully J.A., 1989, A\&A 217, 319

Burgess A., Mason H.E., Tully J.A., 1991, ApJ 376, 803

Burke V.M., 1992, J. Phys. B 25, 4917

Burke P.G., Hibbert A., Robb W.D., 1971, J. Phys. B 4, 153
Burton W.M., Ridgeley, 1970, Sol. Phys. 14, 3

Butler K., Zeippen C.J., 1994, A\&AS 108, 1

Cai W., Pradhan A.K., 1993, A\&AS 88, 329

Christensen B.R., Norcross D.W., Pradhan A.K., 1985, Phys. Rev. A 32, 93

Christensen B.R., Norcross D.W., Pradhan A.K., 1986, Phys. Rev. A 34, 4704

Copeland F.B.M., Reid R.H.G., Keenan F.P., 1996, ADNDT (submitted)

Cornille M., Dubau J., Faucher P., Bely-Dubau F., Blancard C., 1994, A\&AS 105, 77

Cornille M., Dubau J., Loulergue M., Bely-Dubau F., Faucher P., 1992, A\&A 259, 669

Cornille M., Dubau J., Mason H.E., Blancard C., Brown W.A., 1997, A\&A 320, 333

Czyzak S.J., Krueger T.K., 1966, ApJ 144, 381

Dankwort W., Trefftz E., 1978, A\&A 65, 93

Dere K.P., 1978, ApJ 221, 1062

Dere K.P., 1982, Solar Phys. 77, 77

Dere K.P., Cook J.W., 1979, ApJ 229, 772

Dere K.P., Bartoe J.-D.F., Brueckner G.E., 1982, ApJ 259, 366

Dere K.P., Mason H.E., 1981, in Orrall F.Q. (ed.) Active Regions. Colorado Univ. Press, Ch. 6, p. 129

Dere K.P., Mason H.E., Widing K.G., Bhatia A.K., 1979, ApJS 40,341

Doschek G.A., 1997, ApJ 476, 903

Dufton P.L., Kingston A.E., 1989, MNRAS 241, 209

Dufton P.L., Kingston A.E., 1991a, MNRAS 248, 827

Dufton P.L., Kingston A.E., 1991b, Phys. Scr. 43, 386

Dufton P.L., Hibbert A., Kingston A.E., Doschek G.A., 1982, ApJ 257, 338

Dufton P.L., Hibbert A., Kingston A.E., Doschek G.A., 1983a, ApJ 274, 420

Dufton P.L., Kingston A.E., Doyle J.G., Widing K.G., 1983b, MNRAS 205, 81

Dufton P.L., Kingston A.E., Scott N.S., 1983c, J. Phys. B 16, 3053

Dufton P.L., Kingston A.E., Widing K.G., 1990, ApJ 353, 323

Dunn G.H., 1992, Nucl. Fusion Suppl. At. Plasma-Matter. Interaction Data Fusion 2, 25

Dupree A.K., Brickhouse N.S., Doschek G.A., Green J.C., Raymond J.C., 1993, ApJ 418, L41

Dwivedi B.N., 1994, Space Sci. Rev. 65, 289

Edlen B., 1981, Phys. Scripta 23, 1079

Edlen B., 1985, Phys. Scripta 31, 345

Eissner W., Seaton M.J., 1972, J. Phys. B 5, 2187

Eissner W.E., Galavis M.E., Mendoza C., Zeippen, C.T., 1996 (private communication)

Eissner W., Jones M., Nussbaumer H., 1974, Com. Phys. Comm. 8, 270

Fang, et al., 1993, ApJ 413, L141

Fawcett B.C., 1986, ADNDT 35, 203

Fawcett B.C., 1989, ADNDT 43, 71

Fawcett B.C., 1991, ADNDT 47319

Fawcett B., Mason H.E., 1989, ADNDT 43, 245

Fawcett B., Mason H.E., 1991, ADNDT 47, 17

Feldman U., Doschek G.A., 1991, ApJS 75, 925

Feldman U., Doschek G.A., Seely J., 1985, MNRAS 212, 41P

Flower D.R., 1977, A\&A 54, 163

Foster V.J., Mathioudakis M., Keenan F.P., Drake J.J., Widing K.G., 1996, ApJ 473, 560 
Freeman F.F., Jones B.B., 1970, Solar Phys. 15, 288

Froese Fischer C., Liu B., 1986, ADNDT 34, 261

Froese Fischer C., Saha H.P., 1985, Phys. Scripta 32, 181

Gabriel A.H., Jordan C., 1972, in McDaniel E. and McDowell M.C. (eds.) Case Studies in Atomic Collision Physics, Vol. II. North Holland Publ. Co., p. 210

Gaetz T.J., Salpeter E.E., 1983, ApJS 52, 155

Galavis M.E., Eissner W.E., Mendoza C., Zeippen C.J., 1996 (private communication)

Gau J.N., Henry J.W., 1977, Phys. Rev. A 16, 986

Giles K., 1981, MNRAS 195, 63

Grant I.P., McKenzie B.J., Norrington P.H., Mayers D.F., Pyper N.C., 1980, Comp. Phys. Commun. 21, 207

Henry R.J.W., 1993, Rept. Prog. Phys. 56, 327

Hibbert A., 1980, J. Phys. B 13, 1721

Hibbert A., Le Dourneuf M., Mohan M., 1993, ADNDT 53, 23

Ho Y.K., Henry R.J.W., 1983, ApJ 267, 886

Huang K.-N., 1984, ADNDT 30, 313

Hummer D.G., Berrington K.A., Eissner W., Pradhan A.K., Saraph H.E., Tully J.A., 1993, A\&A 279, 298

Itikawa Y., 1991, ADNDT 49, 209

Johansson S., 1978, Phys. Scripta 18, 217

Jupen C., 1984, MNRAS 208, 1P

Jupen C., Isler R.C., Trabert E., 1994, MNRAS 264, 627

Kastner S.O., Neupert W.M., Swartz M., 1974, ApJ 191, 261

Kato T., 1976, ApJS 30, 397

Kato T., 1994, ADNDT 57, 181

Kato T., 1996 ( private communication)

Kato T., Lang J., Berrington K.E., 1990, ADNDT 44, 133

Keenan F.P., 1996, Space Sci. Rev. 75, 537

Keenan F.P., Norrington P.H., 1987, A\&A 181, 370

Keenan F.P., Conlon E.S., Harra L.K., Burke V.M., Widing K.G., 1992, ApJ 385, 381

Keenan F.P., Conlon E.S., Warren G.A., Boone A.W., Norrington P.H., 1993, ApJ 406, 350

Kelly R.L., 1987, J. Phys. Chem. Ref. Data 16, Suppl. 1

Landini M., Monsignori Fossi B.C., 1970, A\&A 6, 468

Landini M., Monsignori Fossi B.C., 1990, A\&AS 82, 229

Lang J., 1994, ADNDT, 57

Lanzafame A.C., 1994, A\&A 287, 972

Lennon D.J., Burke V.M., 1994, A\&AS 103, 273

Lennon D.J., Dufton P.L., Hibbert A., Kingston A.E., 1985, ApJ 294, 200

Loulergue M., Mason H.E., Nussbaumer H., Storey P.J., 1985, A\&A 150, 246

Maran S.P., Robinson R.D., Shore S.N., et al., 1994, ApJ 421, 800

Martin W.C., Zalubas R., 1979, J. Phys. Chem. Ref. Data 8, 817

Martin W.C., Zalubas R., 1980, J. Phys. Chem. Ref. Data 9, 1

Martin W.C., Zalubas R., 1983, J. Phys. Chem. Ref. Data 12, 323

Martin W.C., Zalubas R., Musgrove A., 1990, J. Phys. Chem. Ref. Data 19, 821

Martin I., Karwowski J., Diercksen G.H.F., Barrientos C., 1993, A\&AS 100, 595

Martin W.C., Sugar J., Musgrove A., Dalton G.R., Wiese W.L., Fuhr J.R., 1995, NIST Database for Atomic Spectroscopy, Version 1.0, NIST Standard Reference Database 61

Mason H.E., 1975, MNRAS 170, 651
Mason H.E., 1994, ADNDT 57, 305

Mason H.E., 1996a, in Astrophysics in the EUV, Bowyer S. and Malina R.F. (eds.). Publ. Kluwer Acad. Press, p. 561

Mason H.E., 1996b, unpublished calculations

Mason H.E., Doschek G.A., Feldman U., Bhatia A.K., 1979, A\&A 73,74

Mason H.E., Bhatia, A.K., 1978, MNRAS 184, 423

Mason H.E., Monsignori Fossi B.C., 1994, A\&AR 6, 123

Mason H.E., Storey P.J., 1980, MNRAS 191, 631

McLaughlin B.M., Bell K.L., 1994, ApJS 94, 825

McWhirter R.W.P., 1976, in Atomic and Molecular Processes in Astrophysics, Dalgarno A., Masnou-Seeuws (ed.), p. 187

McWhirter R.W.P., 1994, ADNDT 57, 39

Mendoza C., Zeippen C.J., 1982, MNRAS 198, 127

Mewe R., 1972, Solar Phys. 22, 459

Mewe R., Gronenschild E.H.B.M., 1981, A\&AS 45, 11

Mewe R., Gronenschild E.H.B.M., van den Oord G.H.J., 1985, A\&AS 62, 197

Mewe R., Kaastra J.S., Liedahl D.A., 1993, Legacy 6, 16

Mohan M., Le Dourneuf M., 1990, A\&A 227, 285

Mohan M., Baluja K.L., Hibbert A., 1987, J. Phys. B 20, 2565

Mohan M., Baluja K.L., Hibbert A., 1988, Phys. Scripta 38, 699

Mohan M., Hibbert A., Berrington K.A., Burke P.G., 1990, J. Phys. B 23, 989

Mohan M., Hibbert A., Kingston A.E., 1994, ApJ 434, 389

Monsignori Fossi B.C., Landini M., 1994a, Sol. Phys. 152, 81

Monsignori Fossi B.C., Landini M., 1994b, A\&A 284, 900

Monsignori Fossi B.C., Landini M., 1994c, ADNDT 57, 125

Moore C.E., Tables of Spectra of Hydrogen, Carbon, Nitrogen, and Oxygen Atoms and Ions, Gallagher J.W. (ed.). CRC Press 1993

Muhlethaler H.P., Nussbaumer H., 1976, A\&A 48, 109

Nahar S.N., 1995, A\&A 293, 967

Norrington P.H., Grant I.P., 1987, J. Phys. B 20, 4869

Nussbaumer H., Storey P.J., 1979, A\&A 71, L5

Nussbaumer H., Storey P.J., 1981, A\&A 96, 91

Nussbaumer H., Storey P.J., 1982, A\&A 113, 21

Osterbrock D.E., 1974, Astrophysics of Gaseous Nebulae. W.H. Freeman and Co., San Francisco

Parpia F.A., Johnson W.R., 1972, Phys. Rev. A 26, 1142

Pelan J., Berrington K.A., 1995, A\&AS 110, 209

Phillips K.J.H., Bhatia A.K., Mason H.E., Zarro D.M., 1996, ApJ 466, 549

Pindzola M.S., Griffin D.C., Bottcher C., 1989, Phys. Rev. A 39, 2385

Pradhan A.K., Gallagher J.W., 1992, ADNDT 52, 227

Raju P.K., Dwivedi B.N., 1978, Sol. Phys. 60, 269

Ralchenko Y.V., Vainshtein, L.A., 1996, in UV and X-ray Spectroscopy of Astrophysical and Laboratory Plasmas, Yamashita K. and Watanabe T. (eds.). Univ. Acad. Press, p. 119

Ramsbottom C.A., Bell K.L., Stafford R.P., 1996, ADNDT 63 (in press)

Ramsbottom C.A., Berrington K.A., Bell K.L., 1994, J. Phys. B 27, L811

Ramsbottom C.A., Berrington K.A., Bell K.L., 1995, ADNDT 61,105

Ramsbottom C.A., Berrington K.A., Hibbert A., Bell K.L., 1994, Phys. Scripta 50, 246

Raymond J.C., Smith B.W., 1977, ApJS 35, 419 
Sampson D.H., Zhang H.L., 1992, Phys. Rev. A 45, 1556

Sampson D.H., Goett S.J., Clark R.E.H., 1984, ADNDT 30, 125

Sampson D.H., Weaver G.M., Goett S.J., Zhang H., Clark R.E.H., 1986, ADNDT 35, 223

Sampson D.H., Zhang H.L., Fontes C.J., 1990, ADNDT 44, 209

Sampson D.H., Zhang H.L., Fontes C.J., 1991, ADNDT 48, 25 Sampson D.H., Zhang H.L., Fontes C.J., 1994, ADNDT 57, 97

Saraph H.E., 1972, Comp. Phys. Commun. 3, 256

Saraph H.E., Tully J.A., 1994, A\&AS 107, 29

Savin D.W., Gardner L.D., Reisenfeld D.B., Young A.R., Kohl J.L., 1995, Phys. Rev. A 51, 2162

Stafford R.P., Bell K.L., Hibbert A., 1994, MNRAS 266, 715

Stafford R.P., Hibbert A., Bell K.L., 1993, MNRAS 260, L11

Stern R., Wang E., Bowyer S., 1978, ApJS 37, 195

Storey P.J., Mason H.E., Saraph H.E., 1996, A\&A 309, 677

Sugar J., Corliss C., 1979, J. Phys. Chem. Ref. Data 8, 865

Tayal S.S., 1994, ApJ 426, 449

Tayal S.S., 1995, ApJ 446, 895

Tayal S.S., Henry R.J.W., 1988, ApJ 329, 1023

Tayal S.S., Henry R.J.W., Pradhan A.K., 1987, ApJ 319, 951

Thomas R.J., Neupert W.M., 1994, ApJS 91, 461

Tucker W.H., Koren M., 1971, ApJ 168, 283

Unnikrishnan K., Callaway J., Oza D.H., 1991, Phys. Rev. A 43,5966
Van Regemorter H., 1962, ApJ 136, 906

Vernazza J.E., Mason H.E., 1978, ApJ 226, 720

Widing K.G., 1982, ApJ 258, 835

Widing K.G., 1996 (private communication)

Widing K.G., Feldman U., Bhatia A.K., 1986, ApJ 308, 982

Widing K.G., Sandlin G.D., 1968, ApJ 152, 545

Wiese W.L., Fuhr J.R., 1995, NIST Database for Atomic Spectroscopy, Version 1.0, NIST Standard Reference Database 61

Wiese W.L., Smith M.W. Glennon B.M., Atomic Transition Probabilities, Vol. I, Hydrogen Through Neon, NSRDSNBS 4, 1966

Younger S.M., Wiese W.L., 1979, J. Quant. Spectrosc. Radiat. Transfer 22, 161

Zhang H.L., 1995 (private communication)

Zhang H.L., Pradhan A.K., 1995, A\&A 293, 953

Zhang H.L., Sampson D.H., 1992, ADNDT 52, 143

Zhang H.L., Sampson D.H., 1994, ADNDT 56, 41

Zhang H.L., Sampson D.H., 1995, unpublished calculations

Zhang H.L., Graziani M., Pradhan A.K., 1994, A\&A 283, 319

Zhang H.L., Sampson D.H., Clark R.E.H., Mann J.B., 1987, ADNDT 37, 17

Zhang H.L., Sampson D.H., Fontes C.J., 1990, ADNDT 44, 31 